\title{
Advances in Clinical Cardiology 2020: A Summary of Key Clinical Trials
}

\author{
Aileen Kearney $\cdot$ Katie Linden $\cdot$ Patrick Savage $\cdot$ Ian B. A. Menown
}

Received: February 2, 2021 / Accepted: March 12, 2021 / Published online: April 12, 2021

(C) Crown 2021

\section{ABSTRACT}

Introduction: Despite the challenge of a global pandemic, 2020 has been an invaluable year in cardiology research with numerous important clinical trials published or presented virtually at major international meetings. This article aims to summarise these trials and place them in clinical context.

Methods: The authors reviewed clinical trials presented at major cardiology conferences during 2020 including the American College of Cardiology, European Association for Percutaneous Cardiovascular Interventions, European Society of Cardiology, Transcatheter Cardiovascular Therapeutics and the American Heart Association. Trials with a broad relevance to the cardiology community and those with potential to change current practice were included.

Results: A total of 87 key cardiology clinical trials were identified for inclusion. New interventional and structural cardiology data included trials evaluating bifurcation percutaneous coronary intervention (PCI) techniques, intravascular ultrasound (IVUS)-guided PCI, instantaneous wave-free (iFR) physiological assessment, new generation stents (DynamX

A. Kearney · K. Linden · P. Savage ·

I. B. A. Menown ( $\square)$

Craigavon Cardiac Centre, Southern Health and

Social Care Trust, Craigavon, Northern Ireland, UK

e-mail: ian.menown@southerntrust.hscni.net bioadaptor), transcatheter aortic valve implantation (TAVI) in low-risk patients, and percutaneous mitral or tricuspid valve interventions. Preventative cardiology data included new data with proprotein convertase subtilisin-kexin type 9 (PCSK9) inhibitors (evolocumab and alirocumab), omega-3 supplements, evinacumab and colchicine in the setting of chronic coronary artery disease. Antiplatelet data included trials evaluating both the optimal length of course following PCI and combination of antiplatelet agents and regimes including combination antithrombotic therapies for patients with atrial fibrillation (AF). Heart failure data included the use of sodium-glucose cotransporter 2 (SGLT2) inhibitors (sotagliflozin, empagliflozin and dapagliflozin) and mavacamten in hypertrophic cardiomyopathy. Electrophysiology trials included early rhythm control in AF and screening for AF.

Conclusion: This article presents a summary of key clinical cardiology trials during the past year and should be of relevance to both clinicians and cardiology researchers.

Keywords: Acute coronary syndrome; Anticoagulation; Atrial fibrillation; Cardiology; coronary revascularisation; Heart failure; Lipids; Mitral clip; Myocardial infarction; Transcatheter aortic valve implantation 
Key Summary Points

A concise summary of 87 key cardiology trials presented at major international conferences during 2020.

Trials with clinical relevance to cardiology and the potential to change current practice.

Updates include interventional and structural cardiology, acute coronary syndromes, preventative cardiology, heart failure, electrophysiology and atrial fibrillation.

\section{DIGITAL FEATURES}

This article is published with digital features, including a summary slide, to facilitate understanding of the article. To view digital features for this article go to https://doi.org/10.6084/ m9.figshare.14182505.

\section{INTRODUCTION}

In 2020 many trials with potential to influence clinical practice and future clinical guidelines have been presented at international meetings including the American College of Cardiology (ACC), European Association for Percutaneous Cardiovascular Interventions (EuroPCR), European Society of Cardiology (ESC), Transcatheter Cardiovascular Therapeutics (TCT) and the American Heart Association (AHA). In this article we review key studies within the fields of acute coronary syndrome (ACS), interventional cardiology, heart failure, atrial fibrillation (AF), electrophysiology and cardiovascular prevention.

\section{METHODS}

The results of clinical trials presented at major international cardiology meetings in 2020 were reviewed. In addition to this, a literature search of PubMed, Medline, Cochrane library and Embase was completed including the terms "acute coronary syndrome", "atrial fibrillation", "coronary prevention", "electrophysiology", "heart failure" and "interventional cardiology". Trials were selected based on their relevance to the cardiology community and the potential to change future clinical guidelines or guide further phase 3 research. This article is based on previously completed work and does not involve any new studies of human or animal subjects performed by any of the authors.

\section{Advances in Interventional Cardiology}

During 2020, COVID-19 undoubtedly impacted our clinical practice and decision making regarding percutaneous coronary intervention (PCI). The question arose as to how best to treat ST segment elevation myocardial infarction (STEMI) patients affected by the disease. In a collaborative investigation from 64 sites in the North American COVID-19 STEMI (NACMI) registry, observational data from 594 patients with ST elevation or left bundle branch block and suspected COVID-19 (171 confirmed COVID positive and 423 "persons under interest (PUI)" confirmed COVID negative) were compared vs. a propensity matched historical cohort [1]. COVID-positive patients vs. PUI vs. controls were more likely to be Black, Hispanic, diabetic, have cardiogenic shock pre-PCI, have angiography deferred and have increased hospital mortality and stroke (Table 1). However, in those referred for angiography, door to balloon time was similar and the primary PCI rate was only slightly less.

Data collection is ongoing but these initial findings highlight that COVID-positive patients with STEMI are at higher cardiovascular (CV) risk and should be treated according to usual primary PCI protocols if possible.

The role of routine revascularisation vs. initial medical therapy in stable patients remains controversial despite the ISCHEMIA (International Study of Comparative Health Effectiveness with Medical and Invasive Approaches) trial findings [2]. Bangalore et al. conducted a 
Table 1 Patient characteristics and clinical endpoints in the NACMI registry [1]

\begin{tabular}{lllllc}
\hline & $\begin{array}{l}\text { COVID+ }(\boldsymbol{n}=\mathbf{1 7 1}) \\
(\%)\end{array}$ & $\begin{array}{l}\text { COVID- PUI } \\
(\boldsymbol{n}=\mathbf{4 2 3})(\%)\end{array}$ & $\begin{array}{l}\boldsymbol{p} \text { value } \\
(\mathbf{C}+\text { vs. } \\
\text { PUI })\end{array}$ & $\begin{array}{l}\text { Matched } \\
\text { controls }(\%)\end{array}$ & $\begin{array}{l}\boldsymbol{p} \text { value } \\
(\mathbf{C}+\text { vs. } \\
\text { control })\end{array}$ \\
\hline Black & 27 & 11 & $<0.001$ & 4 & $<0.001$ \\
Hispanic & 24 & 6 & $<0.001$ & 1 & $<0.001$ \\
Diabetes & 44 & 33 & 0.015 & 20 & $<0.001$ \\
Shock pre-PCI & 20 & 14 & 0.074 & 5 & $<0.001$ \\
No angiography & 21 & 5 & $<0.001$ & 0 & $<0.001$ \\
$\begin{array}{l}\text { Primary PCI rates in those } \\
\quad\end{array}$ & 71 & 80 & 0.03 & 81 & 0.15 \\
having angiography & & & & & $<0.001$ \\
Mortality & 32 & 12 & $<0.001$ & 6 & 0.039 \\
\hline
\end{tabular}

meta-analysis of 14 clinical trials involving 14,877 patients with a weighted mean followup of 4.5 years [3]. Notably, all trials excluded left main (LM) disease with most having preserved left ventricular (LV) systolic function and low symptom burden. Routine revascularisation vs. initial medical therapy did not show any difference in mortality (risk ratio [RR] 0.99; 95\% CI [0.90 to 1.09$]$ ) CV death (RR 0.92; 95\% CI [0.80 to 1.06]) or overall MI (RR 0.93; 95\% CI [0.83 to 1.03$])$ but was associated with significant reduction in non-procedural MI (RR 0.76; 95\% CI [0.67 to 0.85]), unstable angina (RR $0.64 ; 95 \%$ CI [0.45 to 0.92]) and greater freedom from angina (RR 1.10; [95\% CI 1.05 to 1.15]). Of note, $31.9 \%$ of patients in the initial medical therapy group eventually underwent revascularisation.

While the ISCHEMIA trial excluded patients with significant LM stenosis ( $\geq 50 \%$ on screening CT), the ISCHEMIA-LM substudy compared the $962(26 \%)$ overall trial participants with intermediate LM stenosis $(25$ to $<50 \%)$ vs. 2737 (74\%) with no significant LM stenosis $(<25 \%)$ [4]. Of interest, intermediate LM was associated with more multivessel coronary disease and $7.0 \%$ of those defined as intermediate LM stenosis by CT were actually found to have severe LM stenosis on invasive angiography. Intermediate LM stenosis was associated with a significant increase in the primary composite endpoint (CV death, MI, resuscitated cardiac arrest, hospitalisation for unstable angina and hospitalisation for heart failure; HR 1.31; 95\% CI [1.06 to 1.61$], p=0.0123$ ) and secondary single endpoints of $\mathrm{CV}$ death, stroke and heart failure. Routine revascularisation vs. initial medical therapy in intermediate LM patients was associated with a significant reduction in non-procedural MI (4.1\% vs. $10.5 \% ; p=0.049)$ and improvement in angina-related quality of life. Thus, intermediate LM may benefit from more intensive prevention therapy and selected intermediate LM patients may benefit from revascularisation.

Several large trials including SYNTAX, EXCEL and NOBLE have sparked much debate as to the best strategy for treating unprotected LM disease [5]. The PRECOMBAT trial (premier of randomised comparison of bypass surgery versus angioplasty using sirolimus-eluting stent in patients with left main coronary artery disease) randomised 600 Korean patients (mean age 62 years) with unprotected LMS disease (mean SYNTAX score 25; mean EuroSCORE 2.6) to intravascular ultrasound (IVUS)-guided PCI with sirolimus-eluting stents $(n=300)$ vs. coronary artery bypass grafting (CABG) $(n=300)$. At 10 years, [6] there was no significant difference in the primary composite 
endpoint of all-cause mortality, MI, stroke or ischaemia-driven revascularisation $(24.7 \%$ vs. $29.8 \%$, HR 1.25 ; $95 \%$ CI 0.93 to $1.69, p=\mathrm{NS}$ ). Repeat revascularisation was more common with PCI $(16.1 \%$ vs. $8 \% ; p<0.05)$ but there was no difference in rates of death, MI or stroke. While the syntax scores were relatively low and the trial not powered for 10-year outcomes, it does support PCI as an alternative to CABG in selected groups.

In patients with multi-vessel disease including a chronic total occlusion (CTO), CABG has historically been the preferred treatment of choice. However, with evolving stent technology and improved CTO techniques we are increasingly seeing PCI employed as an alternative strategy [7]. Xu et al. undertook a singlecentre non-randomised comparison of 5-year outcomes of 2060 patients with at least one CTO undergoing PCI vs. 2264 patients undergoing CABG [8]. In patients who achieved full revascularisation (residual syntax score $\leq 8$ ), there was no significant difference in the primary composite endpoint of death, MI and stroke. However, in patients with incomplete revascularisation PCI was associated with higher rates of the primary endpoint (HR 1.58; $p<0.0001)$ suggesting that operator experience should play a role in strategy selection.

The durability of radial artery versus saphenous vein grafts (SVG) for CABG has been of interest [9]. In a patient-level pooled data metaanalysis, Gaudino et al. compared 10-year outcomes (90\% follow-up) of 534 patients randomised to radial artery grafting vs. 502 receiving SVG. Use of the radial artery was associated with a lower incidence of the primary composite endpoint (death, MI, or repeat revascularisation; 220 vs. 237 events; $p<0.001$ ) and a lower incidence of the composite of death or MI (188 vs. 193 events; $p=0.01)$. Although the sample size was relatively small it does support use of a radial artery graft when technically feasible.

Patients presenting with acute MI undergoing PCI are often anaemic, which in itself is an independent predictor of cardiac events and increased mortality. However, it is unclear if such patients should be transfused as previous trials have yielded conflicting results. REALITY (cost-effectiveness and cost-utility of liberal vs. restrictive red blood cell transfusion strategies in patients with acute myocardial infarction and anaemia) randomised 666 patients (mean age 77 years) to a restrictive (Hb goal $8-10 \mathrm{~g} / \mathrm{dl}$, $n=324$ ) vs. liberal strategy (goal $\mathrm{Hb}>11 \mathrm{~g} / \mathrm{dl}$, $n=342$ ). After 30 days, the restrictive group had a $23 \%$ reduction in the primary endpoint (composite of all-cause death, re-MI, stroke and emergency revascularisation) $(11.0 \%$ vs. $14.0 \%$, HR $0.77,95 \%$ CI 0.50 to $1.18, p<0.05)$ [10] confirming using a lower $\mathrm{Hb}$ target goal of $8-10 \mathrm{~g} / \mathrm{dl}$ is preferable in the acute setting.

The optimum strategy for complex bifurcation PCI remains hotly debated. The DEFINITION II multicentre trial randomised 653 patients with complex coronary bifurcation lesions to a two-stent $(n=331)$ vs. provisional stenting strategy $(n=329)$ [11]. In the two-stent strategy, DK-crush or culotte techniques and IVUS guidance were strongly encouraged. At 12 months, the two-stent strategy was associated with a $48 \%$ reduction in target lesion failure (TLF) defined as the composite cardiac death, target vessel MI or target lesion revascularisation [TLR] $(6.11 \%$ vs. $11.4 \%$, HR $0.52,95 \%$ CI 0.30 to $0.90 ; p=0.019)$ driven by reductions in MI and TLR. This contemporary study suggests that for more complex bifurcations, such as when the side branch lesion length is $>5 \mathrm{~mm}$ or diameter is $\geq 2.75 \mathrm{~mm}$, a twostent technique may be preferable.

The Glagov phenomenon describes the tendency for coronary arteries to positively remodel (expand) in response to atheromatous disease. It has been suggested that the stent strut rigidity may impede this remodelling and thereby contribute to adverse outcomes [12]. The novel DynamX bioadaptor stent, which has a bioresorbable polymer coating allowing the device to uncage and expand over time, enabling adaption to the positive coronary physiology remodelling, was evaluated in a multicentre single arm trial of 50 patients [13]. Acute luminal gain was $1.61 \pm 0.34 \mathrm{~mm}$. At 1 year, IVUS reported the mean device area and mean vessel area increased significantly by $5 \%$ and $3 \%$, respectively, while the mean lumen area was maintained. Stationary optical coherence tomography in seven patients 
demonstrated restoration of cyclic pulsatility. Further studies of this novel device are planned.

While thinner stent struts are associated with improved clinical outcomes in bare metal stents (BMS), reducing strut thickness may affect drug delivery from drug-eluting stents (DES), and there are limited data comparing otherwise similar thin- and thick-strut DES. Thus, 2-year outcomes of 400 patients treated with a thinstrut (84-88 um) cobalt chromium biodegradable polymer biolimus A9-eluting stent (CoCrBP-BES; Biomatrix Alpha) were compared to 857 patients in the LEADERS study patients treated with a stainless steel biodegradable polymer biolimus A9-eluting stent (SS-BP-BES; Biomatrix Flex) [14]. The primary outcome of major adverse cardiac events (MACE) defined as cardiac death, MI or clinically driven target vessel revascularisation (TVR) occurred in the 6.65\% CoCr-BP-BES vs. $13.23 \%$ SS-BP-BES groups (unadjusted HR 0.48 [0.31 to 0.73]; $p=0.0005$ ). While, after adjustment for baseline characteristics and periprocedural MI definitions by propensity analysis and day-3 landmark analysis, the difference in the primary endpoint was no longer significant, there was still a lower incidence of definite/probable stent thrombosis and a lower patient-orientated composite endpoint $(11.7 \%$ vs. $18.4 \%$; HR 0.6 [0.43 to 0.83$]$; $p=0.006)$ supporting the value of a thinner stent strut design in DES.

This concept was also supported by outcomes of BIOFLOW V (safety and effectiveness of the Orsiro sirolimus-eluting coronary stent system in subjects with coronary artery lesions), which randomised 1334 patients, 2:1, to the $60-\mu \mathrm{m}$ Orsiro CoCr-BP-SES vs. the $81-\mu \mathrm{m}$ Xience CoCr-permanent polymer everolimus-eluting stent [15]. At 3 years, Orsiro was associated with lower TLF $(8.2 \%$ vs. $13.6 \%, p=0.002)$, lower stent thrombosis $(0.1 \%$ vs. $1.2 \% ; p=0.018)$ and lower target vessel MI $(2.8 \%$ vs. $0.95 \%$; $p=0.01$ ).

The low-profile Svelte integrated delivery system device (Slender IDS) is a CoCr DES, mounted on a low-compliant balloon with a fixed 0.014-inch guidewire specifically designed for direct stenting. The OPTIMISE trial randomised 1630 patients deemed suitable for direct stenting to the Slender IDS vs. standard therapy (with a rapid exchange Xience or Promus DES [16]. The primary endpoint of TLF at 112 months narrowly missing the prespecified definition of non-inferiority margin $(10.3 \%$ vs. $9.5 \%, p=0.034$ ) although this was confounded by a higher than anticipated incidence of troponin defined peri-procedural MI in both arms. Overall, the Svelte stent performance appeared comparable, but did not offer any advantages over a standard DES design.

Evolving stent technology aiming to accelerate healing may enable a shorter duration of DAPT and hence reduced bleeding risk. The PIONEER III trial randomised 1632 patients (2:1) undergoing PCI to the novel Supreme "healing-targeted" DES with rapid polymer degradation (4-6 weeks) and an ultra-thin permanent eG base layer to promote endothelial migration and protect the underlying $\mathrm{CoCr}$ struts from corrosion and ion leaching vs. a conventional Xience/Promus permanent polymer DES [17]. At 12 months, the healing-targeted DES met the criteria for non-inferiority (TLF $5.4 \%$ vs. $5.1 \%$; $p=$ NS) although there was a trend to higher ischaemia-driven TLR $(2.3 \%$ vs. $1.0 \% ; p=0.06$ ). Further follow-up is planned, which will be of interest.

Similarly, the COBRA REDUCE trial [18] randomised 996 ACS or stable patients undergoing PCI who required anticoagulation but were at high bleeding risk to a novel nanocoated (Polyzene-F) COBRA-PzF stent vs. a standard DES. Those receiving the COBRA-PzF were given a shortened DAPT duration of 14 days followed by oral anticoagulation (OAC) plus aspirin whereas the control group were DAPT for 3-6 months followed by OAC plus aspirin. However, despite facilitating a shorter DAPT duration, the COBRA-PzF arm was not associated with a significant difference in the co-primary outcome of major bleeding $(7.5 \%$ vs. $8.9 \%, p=0.48$ ) and failed to meet the co-primary outcome of non-inferiority for death, MI, stroke or stent thrombosis $(7.7 \%$ vs. $5.2 \%$, $p=0.061)$.

Trimetazidine is a fatty acid oxidation inhibitor that may improve glucose utilisation of ischaemic myocardium. The ATPCI (Efficacy and safety of trimetazidine after percutaneous coronary intervention) trial randomised 6007 
patients with $\mathrm{PCI}<30$ days to oral trimetazidine (35 mg/twice daily) vs. placebo [19]. Trimetazidine did not show any significant reduction in the primary endpoint (CV death, hospitalisation for cardiac events, recurrent/ persistent angina leading to therapy change, or coronary angiography) (23.3\% vs. $24.7 \%$, $p=0.73)$. However, only $<20 \%$ of patients had post-PCI angina, the event rate was $40 \%$ lower than expected, and $<1 / 4$ were female, limiting the assessment of any beneficial effect on microvascular dysfunction.

\section{Intravascular Physiology-Guided Revascularisation}

Unlike the instantaneous free wave ratio (iFR), the fractional flow reserve (FFR) requires pharmacologically induced hyperaemia, which decreases as age increases [20]. It is unclear whether this affects the predictive value of FFR. A pooled analysis [21] of DEFINE FLAIR (functional lesion assessment of intermediate stenosis to guide revascularisation) and iFR SWEDEHEART (Instantaneous wave-free ratio versus fractional flow reserve in patients with stable angina pectoris or acute coronary syndrome trial) trials $(n=4486)$ reported that while there was only a $5 \%$ difference in deferral rates overall ( $45 \%$ with FFR vs. $50 \%$ with iFR), in patients $<60$ years there was a $12 \%$ difference in deferral rates ( $42 \%$ with FFR vs. $54 \%$ with iFR; $p<0.01)$. Thus, FFR might lead to overtreatment in younger patients but underestimation of risk in elderly patients.

It has been shown that anatomical evaluation alone is insufficient to predict the physiological consequences of coronary stenosis [22], which may be in part due to suboptimal imaging. The Optical Coherence Tomography [OCT] Measures Predicting Fractional Flow Reserve (OMEF) study studied 489 patients undergoing FFR and OCT. A strong association between OCT data MLA $<2 \mathrm{~mm}^{2}$ and FFR $<0.8$ was noted $(R=0.525, p<0.001$; AUC 0.80$)$. However, in the 105 patients with negative FFR not undergoing PCI, the presence of OCT MLA $<2 \mathrm{~mm}^{2}$ was associated with higher MACE, highlighting the importance of using a hybrid approach of anatomy and physiology rather than relying solely on one strategy, especially in more complex cases [23].

When assessing intravascular coronary physiology, there may be discordance between epicardial and microvascular compartments, for example an FFR-positive lesion which is coronary flow reserve (CFR) negative. DEFINE flow was a prospective multi-centre study of 430 patients which assessed both FFR and CFR. PCI was undertaken only if both FFR and CFR were positive ( $\leq 0.80$ and $<2.0$, respectively); otherwise, patients were treated medically. At 2 years, MACE at 2 years was numerically higher in the FFR+/CFR - group vs. the FFR-/CFR - group (10.5\% vs. $5.8 \%)$, which did not meet the criteria for non-inferiority [24]. Two-year MACE in the FFR-/CFR+ group was $12.4 \%$. The findings are only hypothesis generating given the small study size but suggest that it may be preferable to consider both FFR and CFR when planning revascularisation.

Diabetic patients are known to have microvascular dysfunction and may have worse outcomes from FFR-negative lesions [25]. The COMBINE (combined techniques improve plaque identification in diabetics) OCT-FFR trial [26] studied prospective diabetic patients with OCT and FFR and compared 98 FFR-negative patients with thin-cap fibroatheromas (TCFA) vs. 292 FFR negative patients with thin-cap fibroatheromas (TCFA). At 18 months, FFRnegative but TCFA-positive patients had a significantly higher incidence of MACE (13.3\% vs. $3.1 \%, \mathrm{HR}=4.7 ; 95 \%$ CI 2.0 to $10.9 ; p=0.0004)$, confirming the importance of such lesions that may benefit from additional medical therapy intensification.

The intravascular physiology definition of successful PCI is typically defined as FFR $>0.9$ but this is not always achieved despite satisfactory angiographic results. The TARGET-FFR single-centre trial randomised 260 patients following angiographically acceptable PCI to further physiology-guided incremental optimisation with further dilatation \pm stenting if clinically appropriate vs. standard care [27]. Core-lab adjudicated results showed that only $32 \%$ of patients with angiographically acceptable PCI initially achieved FFR $>0.9$. Additional physiology-guided optimisation (which was 
considered appropriate in 31\%) was associated with a numerically higher proportion of patients achieving final FFR $>0.9$ (38.1\% vs. $28.1 \%, p=0.099)$ and fewer patients with final FFR $\leq 0.80(18.6 \%$ vs. $29.8 \% ; p=0.045)$.

\section{Intravascular Imaging}

The previously published Lipid-Rich Plaque (LRP) study identified a correlation between lipid core plaque (LCP) and future cardiac events. PROSPECT II [Prospective natural history study using near-infrared spectroscopy (NIRS)-IVUS imaging in patients with acute myocardial infarction] prospectively studied 898 troponin-positive patients following culprit lesion PCI with three vessel NIRS-IVUS (Fig. 1) to identify non-culprit, lipid-rich lesions [28]. At 3.7 years, the primary outcome of MACE was $13.2 \%$ with $8 \%$ arising from a previously nonflow-limiting plaque vs. only $4.2 \%$ arising from the previously treated culprit lesion.

PROSPECT-ABSORB (Providing regional observations to study predictors of events in the coronary tree II study combined with a randomised, controlled, intervention trial) was a substudy of PROSPECT II patients with $\geq 1$ non-flow-limiting lesion but IVUS plaque burden $\geq 65 \%(n=182)$ who were randomised to PCI with an ABSORB bioresorbable scaffold vs. medical therapy alone [29]. The primary endpoint at 25 months of minimum lumen area (MLA) patients was greater in those receiving PCI $\left(6.9\right.$ vs. $\left.3.0 \mathrm{~mm}^{2} ; p<0.0001\right)$. The study was not powered for clinical endpoints. TLF at 24 months did not show any significant difference (Fig. 2). MACE analysis at 4.1 years was numerically although not significantly lower. While intensive lipid lowering, which has been associated with positive remodelling of highrisk plaques [30], remains the treatment of choice for now rather than prophylactic PCI, given the MACE observation, the investigators may consider undertaking a larger randomised trial powered for clinical endpoints.

The ULTIMATE study (Ultrasound-guided versus angiography-guided implantation of drug-eluting stent in all-comers) randomised 1448 patients to IVUS-guided PCI $(n=724)$ vs.

\section{High-Risk Plaque}

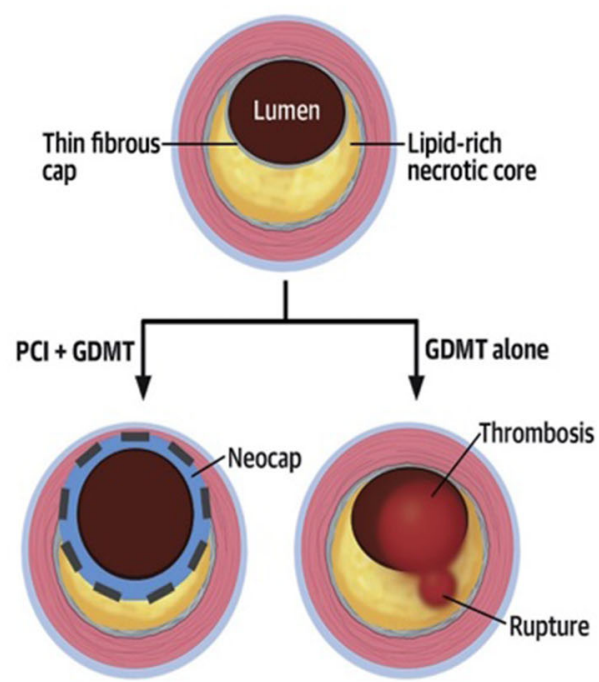

Fig. 1 Central illustration. Conceptual framework for the in vivo detection and focal passivation of vulnerable plaques. PCI percutaneous coronary intervention. GDMT

\section{In Vivo Identification of High-Risk Plaque}

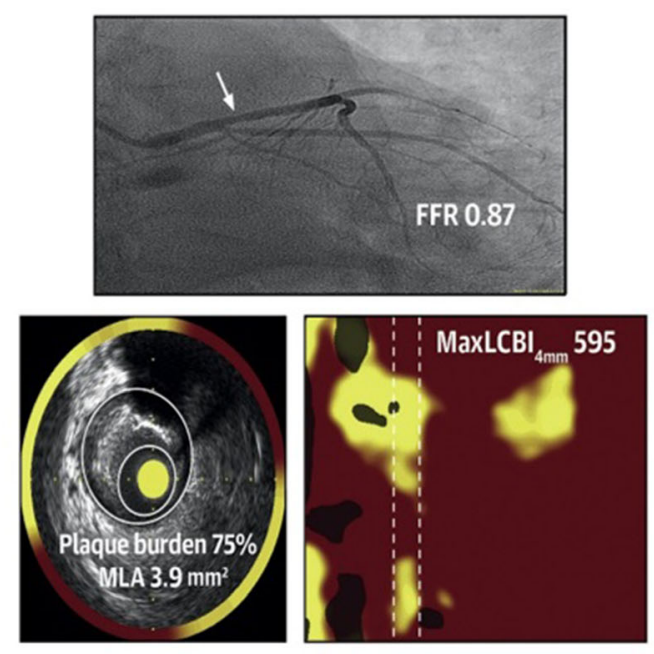

guideline-directed medical therapy (Reproduced with the permission of the Journal of the American College of Cardiology [29]) 

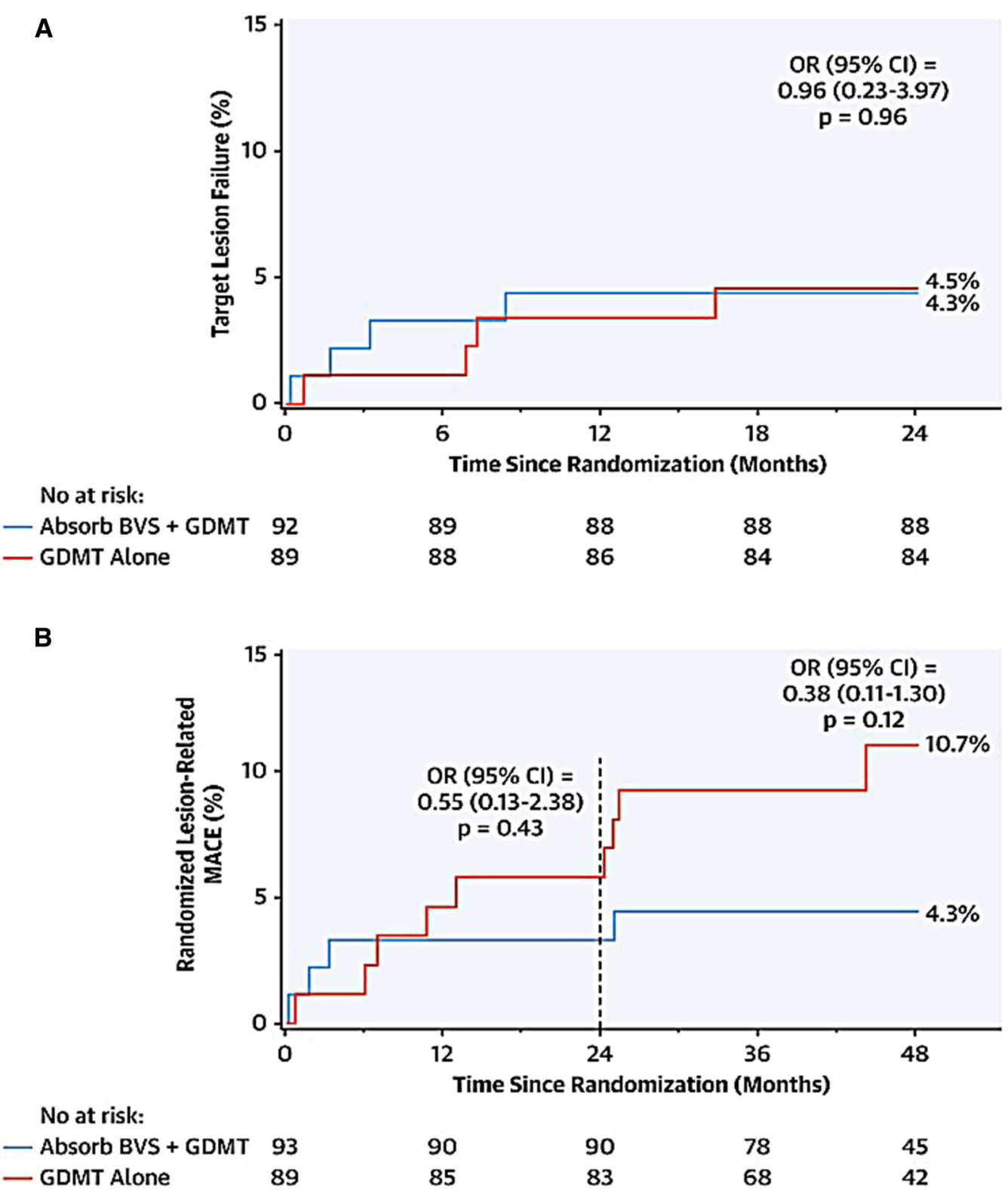

Fig. 2 Kaplan-Meier hazard curves for a target lesion failure, the primary safety outcome measure, through the 24-month follow-up and $\mathbf{b}$ randomised lesion-related major adverse cardiac events (MACE) through the 4-year

angiography only guided PCI $(n=724)$ [31]. At 3-year follow-up, IVUS-guided PCI was associated with a significant reduction in the primary endpoint of TVF $(6.6 \%, 10.7 \% p=0.01)$ and a numerically lower rate of stent thrombosis $(0.1 \%$ vs. $1.1 \%, p=0.10)$. In the IVUS-guided PCI group, those who achieved an "optimal" follow-up in the PROSPECT-ABSORB trial (Reproduced with the permission of the Journal of the American College of Cardiology [29])

result (minimal cross-sectional area $>5.0 \mathrm{~mm}^{2}$, plaque burden at proximal/distal edges $<50 \%$, no edge dissection $>3 \mathrm{~mm}$ involving the media) had a lower rate of TVF vs. those with a sub-optimal IVUS result $(4.2 \%$ vs. $9.2 \%$; $p=0.01)$. 


\section{Advances in Structural Cardiology}

\section{Transcatheter Aortic Valve Implantation}

Transcatheter aortic valve implantation (TAVI) for severe symptomatic aortic stenosis in patients with high or intermediate operative risk is well established. The UK TAVI trial [32] randomised 913 patients (from all 34 UK Hospitals performing TAVI) with severe symptomatic aortic stenosis aged $\geq 80$ years or $\geq 70$ years with intermediate or high surgical risk to TAVI vs. conventional surgical aortic valve replacement (SAVR). A third of patients were $70-79$ years. At 12 months, TAVI was non inferior for the primary endpoint of all-cause mortality $(4.6 \%$ vs. $6.6 \% ; p=0.23)$ and was associated with less major bleeding and shorter hospital stay, but more vascular complications, permanent pacemaker and paravalvular aortic regurgitation. Stroke was similar between treatment groups. Longer follow-up is required to confirm sustained clinical benefit, particularly in younger patients.

The Placement of Aortic Transcatheter Valves (PARTNER) 3 trial previously reported that in patients with severe calcific aortic stenosis and low surgical risk (STS PROM risk score $<4 \%$ ) the Edwards Sapien 3 (a third-generation balloon expandable transfemoral TAVI) was associated with a lower 1 year incidence of death, stroke or rehospitalisation vs. a bioprosthetic SAVR (8.5\% vs. $15.1 \%$; HR 0.54; $95 \%$ CI 0.37 to $0.79 ; p=0.001$ ) [33]. New data presented at ACC 2020 showed that TAVI remained superior for the primary endpoint at 2 years $(11.5 \%$ vs. $17.4 \%)$ although the gap narrowed because of more late deaths and stroke in the TAVI arm between years 1 and 2 [34]. At 2 years, the TAVI arm had an increased incidence of valve thrombosis $(2.6 \%$ vs. $0.7 \% ; p=0.02)$ and higher mean transvalvular gradient (13.6 mmHg vs. $11.8 \mathrm{mmHg} ; p<0.001$ ). While short-term outcomes from Partner 3 are promising, investigators plan follow-up for 10 years to assess valve durability and examine the impact of hypoattenuated leaflet thickening (HALT).

The bicuspid aortic valve has an estimated prevalence of $1 \%$ and is prone to early degeneration often requiring intervention.
Prospective data regarding outcomes of TAVI in low-risk patients with bicuspid aortic valve stenosis are lacking. The Low-Risk Bicuspid Study enrolled 150 patients with severe bicuspid aortic valve stenosis in a prospective, single-arm trial study with inclusion and exclusion criteria developed from the EVOLUT low-risk randomised trial [35]. Device success rate was high at $95.3 \%$. At 30 days, the incidence of all-cause mortality or disabling stroke was $1.3 \%$ (95\% CI 0.3 to $5.3 \%$ ), mean valve gradient of $7.6 \mathrm{mmHg}$, and no patients had any more than mild aortic regurgitation. With the expanding indications for TAVI, adequately powered randomised trials are needed to compare TAVI vs. SAVR for bicuspid aortic stenosis in low-risk patients.

There is a paucity of studies assessing the functional effect of TAVI performed for aortic regurgitation, but it may be offered in experienced centres when surgery is considered too high risk. Graziani et al. reported a retrospective single-centre observational study of 22 patients with severe aortic regurgitation and increased LV dimensions or LV systolic dysfunction treated with TAVI using self-expanding prostheses [36]. The procedural success rate was high $(95.5 \%)$ with mild $(24 \%)$ or no aortic regurgitation (76\%) recorded following TAVI. Haemodynamic parameters including LV end-diastolic pressure and estimated pulmonary artery systolic pressure improved significantly post-TAVI with echocardiographic parameters including LV diastolic diameter and LV mass improving acutely and at short-term follow-up, along with significant reductions in concomitant mitral and tricuspid regurgitation (Table 2). There was a transient, mild LVEF reduction which fully recovered at follow-up. All patients were alive after a mean follow-up time interval of 13 months and $90 \%$ of patients were in New York Heart Association functional class I or II. While these findings are encouraging further evaluation in larger studies is warranted.

The Scope 1 and Scope 2 trials provided two of the few head-to-head comparisons between TAVI valves. Scope 1 randomised patients with severe aortic stenosis and increased surgical risk to the Self-expanding ACURATE neo $(n=372)$ vs. Balloon-expandable Sapien $3(n=367)$ and previously reported 30-day results in which 
Table 2 Comparison of haemodynamic and echocardiographic parameters at baseline and immediately post-TAVI [36]

\begin{tabular}{lccr}
\hline & Baseline & Immediately post TAVI & $\boldsymbol{p}$ value \\
\hline LV end diastolic pressure $(\mathrm{mmHg})$ & 26.2 & 20.1 & 0.012 \\
Estimated pulmonary artery systolic pressure $(\mathrm{mmHg})$ & $48.6 \pm 17.3$ & $32.9 \pm 7.8$ & $<0.001$ \\
LV end-diastolic diameter $(\mathrm{mm})$ & $60.0 \pm 8.0$ & $54.6 \pm 8.1$ & 0.002 \\
LV end-diastolic volume indexed $\left(\mathrm{ml} / \mathrm{m}^{2}\right)$ & $87.1 \pm 30.8$ & $71.4 \pm 25.6$ & $<0.001$ \\
LV mass $\left(\mathrm{g} / \mathrm{m}^{2}\right)$ & $163.2 \pm 58.8$ & $140.2 \pm 45.6$ & 0.004 \\
LV ejection fraction $(\%)$ & $49.1 \pm 13.5$ & $43.3 \pm 13.1$ & 0.008 \\
\hline
\end{tabular}

ACURATE neo failed to meet the criteria for non-inferiority for the primary composite safety and efficacy endpoint at 30 days [37]. New 1-year follow-up data at TCT 2020 [38] reported no significant difference in the composite of allcause mortality or disabling stroke $(12 \%$ vs. 9.4\%; $p>0.05)$ and although the ACURATE neo had a higher incidence of moderate or severe paravalvular regurgitation $(8.9 \%$ vs. $3.6 \%$; $p=0.006)$, it was associated with a lower gradient and a larger effective orifice area. Ongoing follow-up will be important to determine the impact of the differential valve performance on long-term outcomes.

Scope 2 [39] randomised 796 patients $\geq 75$ years with severe symptomatic aortic stenosis and increased surgical risk to the ACURATE neo vs. the Medtronic Core Valve Evolut (both self-expanding, supra-annular valves with porcine pericardial leaflets). ACURATE neo failed to meet the criteria for noninferiority for the composite of death or stroke at 1 year $(15.8 \%$ vs. $13.9 \%$; $p=0.055$ for noninferiority) and was associated with increased rates of cardiac death and paravalvular regurgitation although a lower need for a pacemaker. Longer term follow-up is ongoing.

Current ESC guidelines recommend clopidogrel in addition to aspirin for 3-6 months after TAVI in patients without an indication for OAC [40]. In the open-label POPular TAVI trial (antiplatelet therapy for patients undergoing transcatheter aortic-valve implantation) 665 patients from cohort A were randomised to aspirin alone vs. aspirin plus clopidogrel for 3 months [41]. At 12 months, those receiving aspirin alone had fewer all bleeding events ( $15.1 \%$ vs. $26.6 \%$; risk ratio, $0.57 ; 95 \%$ CI 0.42 to $0.77 ; p=0.001$ ) and fewer non-procedurerelated bleeds, but reassuringly, CV death, stroke or MI remained similar (9.7\% vs. 9.9\%; $p$ for noninferiority $=0.004$ ) and valve function remain similar. The results from this trial support findings from the smaller ARTE (aspirin versus aspirin + clopidogrel following transcatheter aortic valve implantation) trial [42] and may challenge current guideline recommendations with respect to antiplatelets following TAVI.

A significant percentage of patients undergoing TAVI have concomitant $\mathrm{AF}$, requiring OAC. In cohort B of the POPular TAVI trial, 326 patients who underwent TAVI and had a longterm indication for OAC were randomised to OAC alone vs. OAC plus clopidogrel for 3 months [43]. OAC alone was associated with fewer any bleeding events $(21.7 \%$ vs. $34.6 \%$; RR $0.63 ; 95 \%$ CI 0.43 to $0.90 ; p=0.01)$ and fewer non-procedural bleeds $(21.7 \%$ vs. $34 \%$; RR 0.64 ; $95 \%$ CI 0.44 to $0.92 ; p=0.02$ ) and was noninferior with respect to MACE events. Thus, it is preferable to avoid adding clopidogrel to OAC following TAVI, unless there is a specific indication.

Peri-procedural stroke may complicate $2-6 \%$ of TAVI cases. The REFLECT II trial [44] randomised patients $(2: 1)$ to the TriGUARD 3 cerebral embolic protection device vs. usual care. Unfortunately, the sponsor suspended the trial, enrolling only 179 of the 225 planned patients, rendering the trial inconclusive. Based on available data at 30 days, TriGUARD3 vs. 
usual care did not reduce the primary efficacy endpoint (all-cause mortality or stroke). The primary safety endpoint (a composite of allcause mortality, stroke, life-threatening bleeding, stage $2 / 3$ acute kidney injury, coronary artery obstruction requiring intervention, major vascular complication and valve-related dysfunction requiring intervention) met noninferiority vs. an historical performance goal (15.9\% vs. $34.4 \% ; p$ non-inferiority $=0.001$ ) although safety events only actually occurred in $7 \%$ of the randomised usual care group $(p=0.11)$. Overall, the results of REFLECT II are in line with the other evidence suggesting no reduction in stroke rate or mortality with use of embolic protection devices. It remains uncertain whether targeting their use to patients at the highest risk of stroke could be beneficial.

\section{Mitral Valve Interventions}

New technologies for percutaneous therapies for mitral regurgitation (MR) continue to be developed. The PASCAL repair system (Edwards Lifesciences, Irvine, CA) uses two clasps and paddles to achieve coaptation of the mitral valve leaflets, while placing an anatomic spacer to fill the regurgitant orifice area, thus reducing mitral regurgitation. In the multicentre CLASP registry (Edwards PASCAL TrAnScatheter Mitral Valve RePair System Study), 109 patients with symptomatic severe mitral regurgitation (2/3 functional; $1 / 3$ degenerative) underwent PASCAL mitral repair [45] (Fig. 3). At 1 year, the survival rate was high $(92 \%), 88 \%$ were free from heart failure hospitalisation and sustained mitral regurgitation reduction was noted (MR was $\leq 1+$ in $82 \%$ and $\leq 2+$ in $100 \%$ of patients) as were improvements in functional status and quality of life. Pre- and post-medical therapy details were not described, which makes interpreting the observations more difficult, but nonetheless, these results are encouraging and the outcome of the ongoing CLASP IID/IIF (Edwards PASCAL CLASP IID/IIF Pivotal Clinical Trial) comparing the PASCAL mitral repair system to MitraClip (Abbott Vascular) is eagerly awaited [46].

The EXPAND registry evaluated third-generation MitraClip devices with a greater coaptation surface area and ease of use [47] enrolling

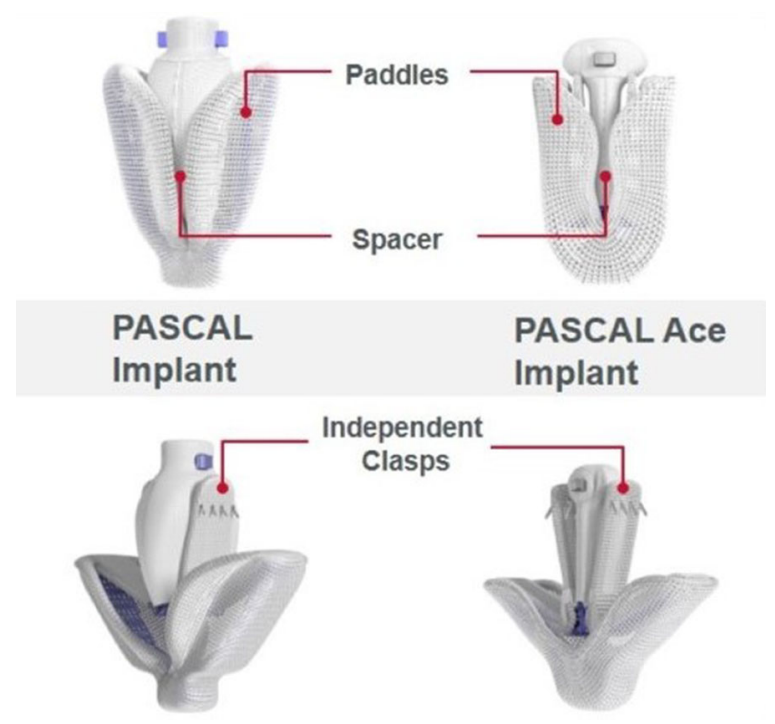

Fig. 3 PASCAL implant and PASCAL Ace implant repair systems with a central spacer, broad contoured paddles and independent clasps for leaflet capture (Reproduced with permission of Edwards Lifesciences Corp., 2021. All rights reserved)

1041 subjects with significant symptomatic MR at 57 centres in the US and Europe. Approximately half had primary MR and half had secondary MR. Higher procedural success rates were noted than in previous registry data [48], which may in part be related to an additional clip size and improvement in the delivery system. At 1 year, mitral regurgitation had reduced to grade $0 / 1$ in $89.2 \%$, mortality was $14 \%$, and 80\% were classified as New York Heart Association Class I or II. Kansas City Cardiomyopathy Questionnaire scores improved by an average of 21.6 points (with similar improvements whether primary and secondary MR).

Mechanical circulatory support can be considered as a bridge to cardiac transplantation in patients with advanced heart failure, but in those with significant concomitant mitral regurgitation there is limited evidence regarding the impact of percutaneous mitral valve intervention. The retrospective multinational MitraBridge registry studied 119 patients with moderate to severe or severe secondary mitral regurgitation treated with Mitraclip as a bridge strategy [49]. Procedural success was achieved in $87.5 \%$ of cases, 30 -day survival was $100 \%$, and 
at 1 year $64 \%$ remained free of death, first rehospitalisation for heart failure, urgent transplantation or LV assist device implantation. Interestingly, at the time of last available follow-up (median 532 days) 23.5\% no longer had an indication for transplantation because of clinical improvement. Further research is needed to explore MitraClip use in this high-risk heart failure population and help identify patients who may derive the greatest benefit.

Transcatheter mitral valve repair (TMVR) requires transseptal access to the left atrium, which in some instances may lead to a persistent iatrogenic atrial septal defect. The singlecentre MITHRAS trial (closure of iatrogenic atrial septal defects following transcatheter mitral valve repair) randomised 80 patients with a persistent iatrogenic atrial septal defect and predominantly left to right shunt resulting in a fraction of pulmonary perfusion [Qp]/fraction of systemic perfusion $[\mathrm{Qs}] \geq 1.3$ detected at 30 days post TMVR to transcatheter closure vs. conservative management [50]. At 5-month follow-up, use of transcatheter closure was not associated with improved 6-min walk test distance, NT-proBNP, heart failure rehospitalisation or mortality and thus does not appear to be indicated for most cases.

\section{Tricuspid Valve Interventions}

Significant tricuspid regurgitation is associated with an increased mortality and heart failure hospitalisation. The Triluminate single-arm trial prospectively evaluated the TriClip (Abbot) device in 85 patients with symptomatic moderate-to-severe tricuspid regurgitation [51]. At 1 year $87.1 \%$ of patients had at least a one-grade sustained reduction in tricuspid regurgitation and $71 \%$ achieved a two-grade reduction although six major adverse events (7.1\%) occurred of which four were $\mathrm{CV}$ deaths. By 12 months, the hospitalisation rate had fallen by $40 \%$ vs. the prior 12 months $(p=0.003)$ and improvement in right ventricular function was also noted. Further randomised trials are required to assess the impact of tricuspid intervention on longer term hard clinical outcomes (Fig. 4).

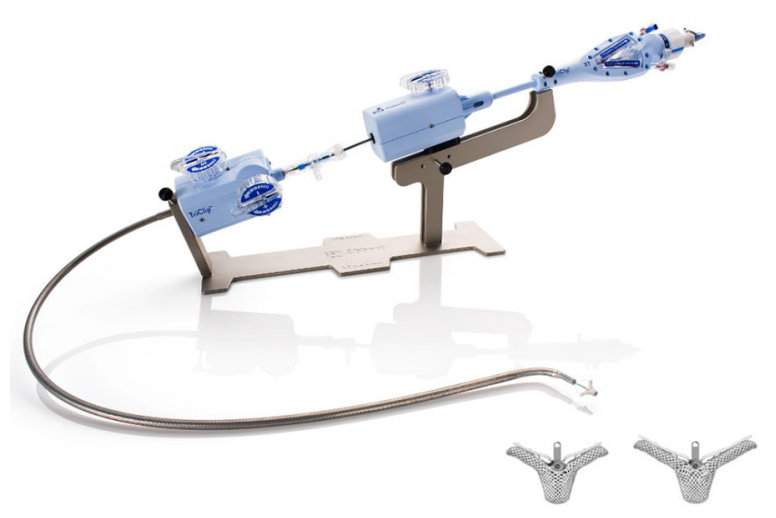

Fig. 4 TriClip $^{\mathrm{TM}}$ delivery system (Reproduced with permission of Abbott, ${ }^{\odot}$ 2020. All rights reserved)

\section{Advances in Cardiovascular Prevention}

\section{LDL Cholesterol}

Monoclonal antibodies that inhibit proprotein convertase subtilisin-kexin type 9 (PCSK9) effectively lower LDL cholesterol (LDLc) levels and reduce the risk of CV events [52-55]. The ODYSSEY HoFH Trial randomised 69 patients with homozygous familial hypercholesterolaemia (FH) to alirocumab $(n=45)$ vs. placebo $(n=24)$. At 12 weeks alirocumab vs. placebo was associated with a marked drop in LDLc from baseline $(-26.9 \% \quad$ vs. $8.6 \% ; \quad p<0.0001)$ apolipoprotein $\mathrm{B}$, non-high-density lipoprotein cholesterol, total cholesterol and lipoprotein(a). This suggests PCSK9 inhibitors offer an effective treatment option for patients with homozygous $\mathrm{FH}$, which has been notoriously difficult to treat. Further studies are required to assess whether this reduction in lipid levels translates to improved $\mathrm{CV}$ outcomes for these patients [56].

Evolocumab initiated during the in-hospital phase of ACS has previously been shown to reduce LDLc effectively by 8 weeks, but the earlier efficacy of PCSK9 inhibitor therapy has been unclear [57]. A new single-centre trial from Japan randomised 102 patients to evolocumab within $24 \mathrm{~h}$ of primary PCI and at 2 weeks $(n=52)$ vs. placebo $(n=50)$ [58]. Those receiving evolocumab showed significantly greater reduction in LDLc by 4 weeks $(-76.1 \%$ vs. $33.1 \% ; p<0.001)$ and all receiving evolocumab achieved LDLc $<1.8 \mathrm{mmol} / \mathrm{l}$ vs. only $27 \%$ of 
the placebo group, thus supporting very early initiation of PCSK9 inhibitor post MI.

Angiopoietin-like 3 (ANGPTL3) is a liverderived circulating factor that inhibits lipoprotein lipase. Loss-of-function ANGPTL3 mutations in humans are associated with low LDLc, low HDLc and low triglycerides. Evinacumab-a human monoclonal antibody against ANGPTL3-was evaluated in a randomised phase 2 study of 272 patients with refractory hypercholesterolaemia (including 116 with heterozygous FH) [59]. At 16 weeks, significant reductions in LDLc were seen with all treatment arms vs. placebo; $p<0.001$ [56.0\% with subcutaneous (s/c) evinacumab $450 \mathrm{mg}$ weekly, $52.9 \%$ with s/c evinacumab $300 \mathrm{mg}$ weekly, $38.5 \%$ with $\mathrm{s} / \mathrm{c}$ evinacumab $300 \mathrm{mg}$ every 2 weeks, $50.5 \%$ with intravenous evinacumab $15 \mathrm{mg} / \mathrm{kg}$ every 4 weeks and $24.2 \%$ with intravenous evinacumab $5 \mathrm{mg} / \mathrm{kg}$ every 4 weeks]. Further trials are planned and evinacumab may have a useful role as an additional option for patients with stubborn LDL cholesterol levels despite more established agents.

Statins, the most commonly used drugs for hypercholesterolaemia, are often stopped because of the reported side effects including muscle aches and weakness. The interesting Self-assessment Method for Statin Side Effects or Nocebo (SAMSON) trial randomised 60 patients, who had previously stopped taking statins because of side effects, to nothing vs. placebo tablet vs. atorvastatin for 1 month duration and then rotated through the other two regimens [60]. Patients recorded symptom intensity daily via a smartphone app. Mean symptom intensity was 8.0 during no-tablet months, 15.4 during placebo months $(p<0.001$ vs. no-tablet months) and 16.3 during statin months $(p<0.001$ vs. no-tablet months; $p=0.39$ vs. placebo) suggesting patients who have discontinued their statin therapy due to reported side effects may well be able to recommence treatment.

\section{Triglycerides}

We have previously discussed the debate regarding the role of omega-3 supplements [2] and findings from the Reduction of CV Events with Icosapent Ethyl-Intervention Trial
(REDUCE-IT), which reported that icosapent ethyl $4 \mathrm{~g}$ daily vs. placebo led to a reduced rate of initial and subsequent CV events [61]. These findings are in contrast to other negative omega- 3 studies and greater than those expected from triglyceride reduction. One difference with REDUCE-IT was the use of high dose EPA only (without DHA), which preserves membrane structure/cholesterol distribution and reduces lipid oxidation. A new analysis presented at ACC 2020 compared on-treatment serum EPA levels with observed CV events and showed a consistent relationship between higher serum EPA levels (200-300 mcg/ml) and reduced CV events both for primary and secondary endpoints supporting the concept that high-dose EPA is needed to optimise event reduction [62].

In contrast, the outcomes study to assess statin residual risk reduction with EpaNova in high CV risk patients with hypertriglyceridaemia (STRENGTH) trial, which randomised 13,078 patients at high CV risk to a high-dose carboxylic acid (CA) formulation of EPA plus DHA vs. corn oil, was halted early in January 2020 as an interim analysis indicated benefit was unlikely [63]. At the time of study discontinuation, omega-3 CA showed no significant reduction in the primary composite endpoint of $\mathrm{CV}$ death, nonfatal MI, nonfatal stroke, coronary revascularisation or unstable angina requiring hospitalisation $(12.0 \%$ vs. $12.2 \%$; $p=0.84$ ) but a greater rate of gastrointestinal adverse events.

Similarly, the Omega-3 Fatty Acids in Elderly with Myocardial Infarction (OMEMI) trial [64], which randomised 1027 elderly patients to $1.8 \mathrm{~g}$ n-3 PUFA (930 mg EPA and $660 \mathrm{mg}$ DHA) vs. corn oil, reported no significant reduction in the composite primary endpoint of non-fatal MI, unscheduled revascularisation, stroke, allcause death and heart failure hospitalisation at 2 years $(21.4 \%$ vs. $20.0 \% ; p=0.60)$.

In summary, there may be a place for icosapent ethyl in secondary prevention but given the disappointing findings with EPA/DHA combinations, further work with high dose EPA is required to confirm clinical benefit and help clarify potential mechanisms of benefit. 

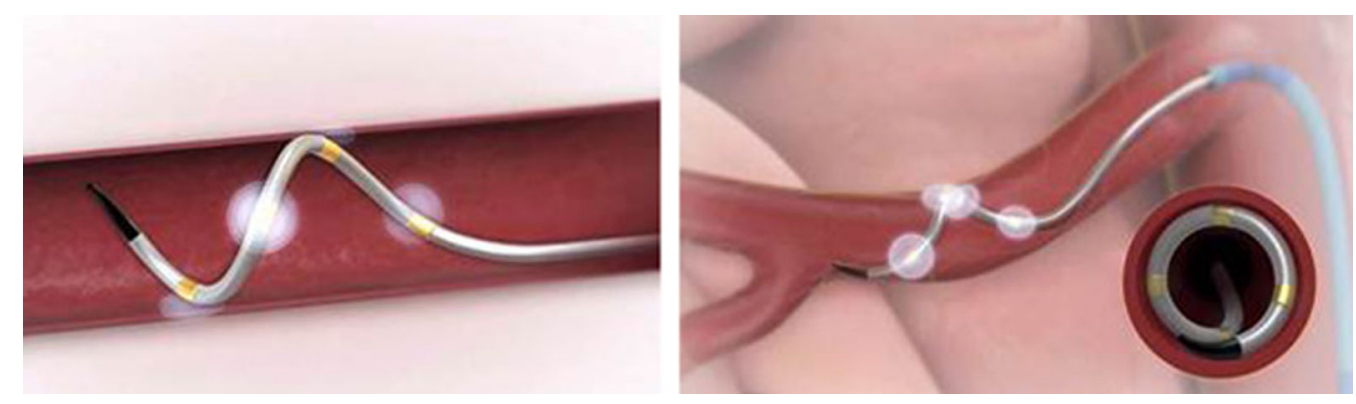

Fig. 5 Symplicity Spyral catheter (Used with the permission of Medtronic. ${ }^{\circledR} 2013$ Medtronic)

\section{Hypertension}

The optimal role of renal artery denervation (RDN) for managing uncontrolled hypertension remains unclear although initial concerns regarding lack of benefit in SYMPLICITY HTN-3 were assuaged by positive data from subsequent sham-controlled trials [65]. New 3-year data from the ongoing global SYMPLICITY registry reported a mean reduction in 24-h systolic blood pressure (SBP) of $-8.9 \pm 20.1 \mathrm{mmHg}$ $(p<0.0001)$ for the overall cohort and $\mathrm{a}-10.4 \pm 21.0 \mathrm{mmHg}(p<0.0001)$ reduction for patients with resistant hypertension [66] (Fig. 5). Following on from an earlier proof-ofconcept pilot trial, the SPYRAL HTN-OFF MED pivotal trial [67] randomised 331 patients with hypertension (SBP 150 to $<180 \mathrm{mmHg}$ off medication) to $\operatorname{RDN}(n=166)$ vs. a sham procedure $(n=165)$. At 3 months, RDN was associated with a significant reduction in the primary endpoint (baseline-adjusted 24-h SBP $-3.9 \mathrm{mmHg} \quad[95 \% \quad \mathrm{CI} \quad-6.2$ to $-1.6 \mathrm{mmHg}]$ ) and in baseline-adjusted office SBP $(-6.5 \mathrm{mmHg}$ [95\% CI -9.6 to -3.5$])$. Of note, $17 \%$ of patients in the control (sham) arm had to drop out of the trial to commence medication as their BP had risen $>180 \mathrm{mmHg}$. The trial has now moved into a second phase with participants being commenced on antihypertensive therapy and up-titrated to target to evaluate whether RDN patients require less or no medication.

New evidence from the Blood Pressure-Lowering Treatment Trialists' Collaboration (BPLTTC) - a meta-analysis of 48 studies including 348,854 participants [68]-reported that every $5 \mathrm{mmHg}$ reduction in SBP was associated with around a $10 \%$ reduction in major
Table 3 Effect on major $\mathrm{CV}$ events for each $5 \mathrm{mmHg}$ reduction in SBP by entry SBP

\begin{tabular}{lll}
\hline $\begin{array}{l}\text { Systolic blood } \\
\text { pressure at } \\
\text { entry } \\
(\mathbf{m m H g})\end{array}$ & $\begin{array}{l}\text { Hazard ratio for } \\
\text { CVD events } \\
\text { patients with } \\
\text { prior CV events } \\
(\boldsymbol{n}=\mathbf{1 6 0 , 2 7 1})\end{array}$ & $\begin{array}{l}\text { Hazard ratio for } \\
\text { CVD events } \\
\text { patients without } \\
\text { prior CV events } \\
(\boldsymbol{n}=\mathbf{1 8 8 , 5 8 3})\end{array}$ \\
\hline$<120$ & 0.82 & 0.80 \\
$120-129$ & 0.86 & 0.96 \\
$130-139$ & 0.99 & 0.90 \\
$140-149$ & 0.89 & 0.95 \\
$150-159$ & 0.89 & 0.88 \\
$160-169$ & 0.83 & 0.88 \\
$>170$ & 0.90 & 0.89 \\
\hline
\end{tabular}

$\mathrm{CV}$ events. The risks of stroke, ischaemic heart disease, heart failure and death from CVD were reduced by $13 \%, 7 \%, 14 \%$ and $5 \%$, respectively (Table 3). Benefits were seen across consistently across the full range of entry SBP and present in patients with or without prior CV disease. This study suggests use of BP-lowering therapy might be beneficial even in patients with SBP conventionally considered as within the normal range.

\section{Vascular Inflammation}

Inflammation plays a central role in CV pathogenesis [65] disease and the role of the anti-inflammatory drug colchicine has previously been shown to be beneficial as secondary prevention 
in patient with recent MI [69]. The Low-Dose Colchicine for Secondary Prevention of CV Disease (LoDoCo2) randomised 5522 patients with chronic coronary disease to colchicine $500 \mathrm{mg}$ od $(n=2762)$ vs. placebo $(n=2760)$ [70]. After an average follow-up of 28.6 months, colchicine was associated with a $31 \%$ reduction in the composite primary endpoint of $\mathrm{CV}$ death, spontaneous MI, ischaemic stroke and ischaemia-driven coronary revascularisation $(6.8 \%$ vs. 9.6\%; HR 0.69; [95\% CI 0.57 to 0.83 ; $p<0.001)$. The study was limited by absence of CRP data, relatively few women and recruitment of a relatively high-risk group (84\% prior ACS; $84 \%$ prior revascularisation; 3\% annual event rate). As expected, colchicine was associated with a higher incidence of GI intolerance. However, LoDoCo2 suggests that for patients with chronic coronary disease, colchicine may be useful as additional therapy on top of conventional secondary prevention, for those who can tolerate it. Further studies would be useful to identify which patient subgroups would be most likely to benefit.

\section{Primary Prevention}

The International Polycap Study (TIPS-3) [71] randomised 5713 individuals without existing $\mathrm{CV}$ disease and an intermediate risk of future events to a polypill (containing simvastatin $40 \mathrm{mg}$, atenolol $100 \mathrm{mg}$, hydrochlorothiazide $25 \mathrm{mg}$ and ramipril $10 \mathrm{mg}$ ) vs. placebo and also, in $2 \times 2$ design, to aspirin $75 \mathrm{mg}$ vs. placebo. The polypill, which was associated with a $0.49 \mathrm{mmol} / \mathrm{l}$ reduction in LDLc and $5.8 \mathrm{mmHg}$ reduction in SBP, led to a $21 \%$ numerical reduction (just missing statistical significance) in the composite primary outcome of $\mathrm{CV}$ death, MI, stroke, resuscitated cardiac arrest, heart failure or revascularisation $(4.4 \%$ vs. $5.5 \%$; HR $0.79 ; 95 \%$ CI 0.63 to 1.00$)$. Aspirin vs. placebo led to a $14 \%$ numerical reduction in the primary outcome (HR 0.86; 95\% CI 0.67 to 1.10 ). Although previous studies have failed to show a benefit for aspirin for primary prevention [72], interestingly the use of both the polypill plus aspirin together showed a significant 31\% reduction in the primary outcome (HR 0.69; CI 0.50 to 0.97$)$.
Despite widespread take-up, there are few data available on the utility and safety of e-cigarettes. The Evaluating the Efficacy of E-cigarette Use for Smoking Cessation (E3) trial randomised 376 smokers to nicotine e-cigarette, non-nicotine e-cigarettes or no e-cigarettes $(n=121)$ for 12 weeks. All groups received individual counselling [73]. Successful abstinence at 12 weeks was significantly higher with nicotine e-cigarettes vs. no e-cigarettes $(21.9 \%$ vs. $9.1 \%$; risk difference 12.8 [95\% CI 4.0 to 21.6]) but numerically higher with non-nicotine e-cigarettes vs. no e-cigarettes $(17.3 \%$ vs. 9.1\%; risk difference, 8.2 [95\% CI -0.1 to 16.6]). Although promising, further long-term safety data are required before e-cigarettes can be recommended more widely.

\section{Beta-Blockers for Secondary Prevention}

Current recommendations for ongoing betablocker use in secondary prevention are based on relatively old studies. New data from a large Danish registry studied 30,177 patients at 3 months post MI, all with normal LVEF and treated by PCI, identifying those prescribed a beta-blocker $(n=24,770)$ or not $(n=5407)$ [74]. At 3 years, use of a beta-blocker was not associated with any reduction in the composite of $\mathrm{CV}$ death, recurrent MI, heart failure, stroke, angina or a cardiac procedure $(22.9 \%$ vs. $21.6 \%)$ or any reduction in recurrent $\mathrm{MI}$ (6.7\% in both groups) suggesting that beta-blockers may not be required as soon as 3 months post-MI.

\section{Coronavirus}

During the coronavirus pandemic in 2020 there have been conflicting reports regarding the presence of the SARS-CoV-2 virus, which enters cells via ACE receptors, and the safety of cardiac medications including ACE inhibitors and ARBs. The BRACE CORONA trial randomised 659 patients, with a confirmed diagnosis of COVID-19, to either continue or temporarily stop their usual ACE inhibitor/ARB for 30 days [75]. Continuing therapy was not associated with a difference in the primary outcome (number of days alive and out of hospital 21.9 vs. 22.9 , HR $0.95,95 \%$ CI 0.90 to $1.01, p=0.09$ ) or in 30 -day mortality rate $(2.8 \%$ vs. $2.7 \%)$ 
supporting ongoing use of ACE inhibitors and ARB patients with mild to moderate COVID-19 infections.

\section{Advances in ACS and Antithrombotic Therapy}

In patients with non-valvular AF undergoing PCI, dual therapy (OAC plus a single antiplatelet) has consistently been associated with a lower risk of major haemorrhage compared with triple therapy (OAC and dual antiplatelet therapy) but a signal to early ischaemic risk is recognised [76]. The AUGUSTUS trial (antithrombotic therapy after acute coronary syndrome or PCI in atrial fibrillation) (discussed previously [56]) reported less bleeding with apixaban vs. vitamin $\mathrm{K}$ antagonist (VKA) and with placebo vs. aspirin at 6 months but the number of ischaemic events was numerically higher with placebo. In a new post hoc analysis [77], use of aspirin vs. placebo immediately and for up to 30 days resulted in an equal trade-off between an increase in severe bleeding (absolute risk $0.97 \%$ [95\% CI 0.23 to 1.70$]$ ) and a reduction in severe ischaemic events (absolute risk $-0.91 \%$ [95\% CI -1.74 to -0.08$]$ ). After 30 days, aspirin vs. placebo continued to increase bleeding (absolute risk 1.25\% [95\% CI 0.23 to 2.27]) without significantly reducing ischaemic events (absolute risk - 0.17\% [95\% CI -1.33 to 0.98$]$ ). Thus, use of aspirin for up to 30 days may be appropriate in patients at high ischaemic risk, but best avoided in patients at high bleeding risk. Of note, the majority $(92.6 \%)$ of patients in this study received clopidogrel and 30\% had loss of function (LOF) mutations in the CYP2C19 gene; thus, it is unclear whether whether other P2Y12 antiplatelets would have conferred superior ischaemic protection without increasing bleeding risk.

While LOF mutations in the CYP2C19 gene responsible for metabolising clopidogrel into its active metabolites are common, in clinical practice genotyping is not routinely performed prior to prescription. TAILOR-PCI [78] randomised 5302 patients undergoing PCI to genotype-guided therapy [CYP2C19 LOF carriers were given ticagrelor; noncarriers were given clopidogrel] $(n=2652)$ vs. standard therapy with clopidogrel $(n=2650)$ therapy. At 12 months, use of genotyping did not significantly reduce the primary endpoint of $\mathrm{CV}$ death, MI, stroke, stent thrombosis and severe recurrent ischaemia $(4.4 \%$ vs. $5.3 \%, \mathrm{HR}, 0.84$ [95\% CI 0.65 to 1.07 ]; $p=0.16$ ). This provides confidence that routine genotyping prior to the prescription of clopidogrel is not required in routine clinical practice. In keeping with previous data, use of ticagrelor in CYP2C19 LOF carriers vs. clopidogrel control was associated with a strong trend to reduction in the primary endpoint (4.0\% vs. 5.9\%; HR 0.66; 95\% CI 0.43 to $1.02, p=0.06$ ) although it did not quite achieve significance.

Given the bleeding risk with longer DAPT duration, the TICO study (Effect of ticagrelor monotherapy vs. ticagrelor with aspirin on major bleeding and CV events in patients with acute coronary syndrome) randomised 3056 patients with ACS undergoing PCI with the Orsiro stent to shorter duration (3 months) DAPT then ticagrelor monotherapy vs. 12-months DAPT [79]. At 12 months, shorter duration DAPT was associated with a $2 \%$ $(p=0.01)$ reduction in the primary endpoint of net adverse clinical events (NACE), driven by a reduction in major bleeding ( $1.7 \%$ vs. $3.0 \%$; HR 0.56 ; $95 \%$ CI 0.34 to 0.91 ) without a significant difference in thrombosis rate $(0.4 \%$ vs. $0.3 \%$, $p=0.53)$. While shorter duration DAPT appears reasonable, it is unclear if similar outcomes would be achieved if the DAPT was switched to aspirin rather than ticagrelor monotherapy.

While shorter duration DAPT may be preferable in patients at high bleeding risk [80-82], it has been unclear whether this approach was appropriate in patients at high ischaemic risk. TWILIGHT (ticagrelor with aspirin or alone in high-risk patients after coronary intervention) randomised 7119 patients undergoing PCI and with $\geq 1$ high-risk feature of ischaemia or bleeding to shorter duration DAPT (aspirin and ticagrelor for 3 months) followed by ticagrelor monotherapy $(n=3555)$ vs. continued DAPT for 12 months $(n=3564)$. At 12 months, short duration DAPT then ticagrelor monotherapy was associated with a lower risk of BARC 2, 3 or 5 bleeding 
(4.0\% vs. $7.1 \%$; HR 0.56 ; $95 \%$ CI 0.45 to 0.68 ) but no difference in all-cause mortality, MI or stroke $(3.9 \%$ vs. $3.9 \%, p<0.001$ for noninferiority) or stent thrombosis (0.4 vs. 0.6\%) [83]. The TWILIGHT-DM substudy confirmed these findings in patients with diabetes $(n=2620)$ where shorter duration DAPT then ticagrelor monotherapy was associated with a 2.2\% reduction in BARC 2,3 or 5 bleeding $(4.5 \%$ vs. $6.7 \%$; HR $0.65,95 \%$ CI 0.19 to $0.63, p=0.001$ ) vs. DAPT but no difference in the composite of death, MI, or stroke $(4.6 \%$ vs. $5.9 \%$; HR 0.77 , $95 \%$ CI 0.55 to 1.09 ; $p=0.14$ ) [84]. The TWILIGHT-COMPLEX substudy in patients who underwent complex PCI $(n=2342)$ similarly demonstrated shorter duration DAPT then ticagrelor monotherapy was associated with a significant reduction in BARC 2,3 or 5 bleeding ( $4.2 \%$ vs. $7.7 \%$, HR $0.54,95 \%$ CI 0.38 to 0.76 ) but no difference in the death, MI or stroke (3.8\% vs. $4.9 \%$, HR $0.77,95 \%$ CI 0.52 to 1.15 , $p=0.13)$ [85].

Dose adjustment with another potent P2Y12, prasugrel, was tested in HOST-REDUCE-POLYTECH-ACS (harmonising optimal strategy for treatment of coronary artery diseases-comparison of REDUCtion of prasugrEl dose or POLYmer TECHnology in ACS patients), which enrolled 2338 Asian patients who had undergone PCI for ACS. After 1 month of standard dose DAPT (prasugrel $10 \mathrm{mg}$ plus aspirin), patients were randomised to reduced dose prasugrel $5 \mathrm{mg}$ plus aspirin vs. ongoing standard dose DAPT [86]. At 1 year, those in the reduced dose prasugrel arm showed a $30 \%$ reduction in the primary endpoint of net adverse events (death, MI, stent thrombosis, clinically driven revascularisation, stroke or BARC 2 + bleeding) (7.2\% vs. $10.1 \%$, HR $0.70,95 \%$ CI 0.52 to 0.92 , $p=0.012)$, no significant difference in ischaemic events (HR 0.76 [0.40 to 1.45]; $p=0.40$ ) but significantly lower bleeding (HR 0.48 [0.32 to 0.73 ]; $p=0.0007$ ). While this suggests dose reduction of prasugrel after 1 month is preferable in an Asian population (of mean weight $72 \mathrm{~kg}$ ) it is unclear if it can be directly translated to Western populations with higher BMI.

While use of intensive P2Y12 inhibition is superior to clopidogrel in the setting of ACS [87], the benefits are less clear in elective PCI
[88]. ALPHEUS (assessment of loading with the P2Y12 inhibitor ticagrelor or clopidogrel to halt ischaemic events in patients undergoing elective coronary stenting) [89] randomised 1883 patients undergoing elective PCI to ticagrelor vs. clopidogrel loading. Ticagrelor was not associated with any difference in the primary outcome (PCI-related type $4 \mathrm{MI}$ or major myocardial injury at $48 \mathrm{~h})(35 \%$ vs. $36 \%$; OR $0.97,95 \%$ CI 0.80 to $1.17 ; p=0.75$ ) or major bleeding, but was associated with increased minor bleeding ( $11 \%$ vs. $8 \%$; OR $1.54,95 \%$ CI 1.12 to $2.11 ; p=0.0070$ ) supporting clopidogrel remaining the standard of care in elective PCI.

Even in the ACS setting, it is unclear whether the risk:benefit of intensive P2Y12 inhibition becomes unfavourable above a certain age. Although sub-analysis of PLATO (prospective randomised PLATelet inhibition and patient outcomes) did not show any heterogenicity in clinical benefit for ticagrelor in patients $\geq 75$ vs. $<75$ years with respect to primary efficacy and safety endpoints, it did report an excess of non-CABG-related bleeding and data were limited in the very elderly [90]. New data from the SWEDEHEART registry (Swedish web-system for enhancement and development of evidencebased care in heart disease evaluated according to recommended therapies) reported that in patients $\geq 80$ years $(n=14,005)$ discharged following MI from 2010 and 2017 [91], use of ticagrelor $(39.8 \%)$ vs. clopidogrel $(60.2 \%)$ was associated with a similar primary ischaemic outcome (HR 0.97 [95\% CI 0.88 to 1.06 ]), a 17\% higher risk of death (HR 1.17 [1.03 to 1.32]) and $48 \%$ higher risk of bleeding (HR 1.48 [1.25 to $1.76]$ ), but $20 \%$ lower risk of MI (HR 0.80 [0.70 to 0.92$])$ and $28 \%$ lower risk of stroke (0.72 [0.56 to 0.93$])$. Interestingly, at $<80$ years of age this risk:benefit ratio shifted in favour of ticagrelor with a $15 \%$ lower risk of death (0.85 [0.76 to $0.96])$ and $17 \%$ lower rate of ischaemia (0.83 [0.77 to 0.89]). Overall, this supports use of ticagrelor post-ACS in those $<80$ years but suggests clopidogrel may be preferable at $\geq 80$ years although a prospective randomised trial would be needed to definitively address the question.

Optimal timing of P2Y12 inhibition in ACS is incompletely understood. Downstream (no 
pre-treatment) therapy is advised with prasugrel although the upstream (pre-treatment) strategy is common practice with ticagrelor. DUBIUS (downstream versus upstream strategy for the administration of P2Y12 receptor blockers in non-ST elevated acute coronary syndromes with initial invasive indication) randomised 1449 patients to downstream (ticagrelor or prasugrel) vs. upstream (ticagrelor only) treatment [92]. The trial was stopped early at interim analysis because of futility with no difference seen in the primary endpoint of vascular death, MI, stroke or BARC $3-5$ bleeding at 30 days (2.9\% vs. $3.3 \%$, ARR\% - 0.46 [- 2.90; 1.90]). Although stopped early, DUBIUS suggests no practical difference in outcome between up- or downstream strategies.

Crushing of ticagrelor or prasugrel in STEMI results in faster drug absorption and more rapid platelet inhibition [93, 94] but the clinical benefit has been unclear. COMPARE-CRUSH randomised 727 patients undergoing primary PCI to crushed $(n=369)$ vs. integral $(n=358)$ prasugrel $60 \mathrm{mg}$ tablets [95]. While crushed prasugrel was associated with enhanced platelet inhibition (P2Y12 reactivity unit 192 vs. 227; $p \leq 0.01$ ) there was no significant difference in the primary endpoint of culprit artery TIMI 3 flow $(31.0 \%$ vs. $32.7 \%, p=0.64)$, ST resolution $1 \mathrm{~h}$ post-PCI $(59.9 \%$ vs. $57.3 \%, p=0.55)$ or stent thrombosis $(3.3 \%$ vs. $3.9 \%, p=1.0)$. This suggests it is unnecessary to crush prasugrel prior to PCI (although the study was underpowered for stent thrombosis).

Given that opioids may delay absorption of P2Y12 inhibitors [96], the ON-TIME3 trial (opioids and crushed ticagrelor in myocardial infarction evaluation) randomised 195 patients with STEMI, pre-treated with crushed ticagrelor, to intravenous (IV) paracetamol (acetaminophen) $(n=98)$ vs. IV fentanyl $(n=97)$ [97]. Use of paracetamol resulted in similar pain relief and higher levels of systemic ticagrelor at the start of primary PCI $(151 \mathrm{ng} / \mathrm{ml}$ vs. $60 \mathrm{ng} /$ $\mathrm{ml} ; p=0.007)$ but no significant difference in the primary endpoint of platelet inhibition (P2Y12 reactivity unit 104 vs. $175 ; p=0.18$ ). While the primary endpoint was not met, the trial does support consideration of IV paracetamol analgesia in the setting of primary PCI.
Direct oral anticoagulants (DOACs) are licenced for non-valvular AF, with superiority vs. vitamin $\mathrm{K}$ antagonist (VKA) demonstrated in previous trials $[98,99]$. ESC guidelines allow for DOAC use in valvular AF (aside from moderate to severe mitral stenosis and mechanical prosthetic heart valves) although this is based on meta-analysis of prior trial data rather than prospective data. The RIVER trial randomised 1005 patients with a bioprosthetic mitral valve and $\mathrm{AF} /$ flutter to rivaroxaban vs. VKA [100]. Rivaroxaban was non-inferior $(p<0.0001)$ in meeting the primary outcome (death, major adverse cardiac events, major bleeding) with fewer CVD or thromboembolic events (3.4\% vs. $5.1 \%$, HR $0.65,95 \%$ CI 0.35 to 1.20 ) and fewer strokes $0.6 \%$ vs. $2.4 \%$ (HR $0.25,95 \%$ CI 0.07 to $0.88)$. Although a relatively young population with a higher incidence of rheumatic heart disease than typical European/US populations, it supports the guideline position of DOAC use in such patients.

MI with non-obstructive coronary arteries (MINOCA) is increasingly recognised, affecting predominantly females, but optimal assessement is unclear [101]. In a prospective study of 301 women with a clinical diagnosis of MI, 170 were diagnosed with MINOCA of whom $145 / 170$ had adequate OCT image quality for analysis and 116/145 also had CMR imaging within 1 week [102]. OCT showed a definite or possible culprit lesion in $46 \%(67 / 145)$, most commonly plaque rupture, intra-plaque cavity or layered plaque. CMR was abnormal in $74.1 \%$ (ischaemic in 62/116 and non-ischaemic in 24/116). A combined OCT and CMR approach was most useful for identifying a cause for MINOCA, which was identified in $84.5 \%$ (around 3/4 ischaemic and 1/4 non-ischaemic including myocarditis, takotsubo syndrome and non-ischaemic cardiomyopathy). While the cost of such an approach is relatively high, the results are very important highlighting a much higher incidence of ischaemic cause than perhaps otherwise expected with implications for ongoing risk factor management.

The value of computerised tomography coronary angiography (CTCA) is well established in stable chest pain and has previously been found useful in low to intermediate risk 
patients presenting with acute chest pain [103]. The RAPID-CTCA trial (rapid assessment of potential ischaemic heart disease with CTCA) randomised 1748 patients with suspected ACS (acute chest pain plus elevated troponin 57\%, abnormal ECG 61\%) to CTCA vs. usual care [104]. CTCA coronary findings were normal in $23 \%$, nonobstructive in $29 \%$ and obstructive in $47 \%$. CTCA patients were less likely to undergo invasive angiography (adjusted HR 0.81; 95\% CI 0.72 to 0.92 ) but equally likely to undergo revascularisation and the primary outcome (death or MI at 1 year) was similar $(5.8 \%$ vs. $6.1 \% ; p=0.65$ ) However, CTCA was associated with longer hospital stay and higher cost and thus does not appear to have a role in routine evaluation of such patients.

In non-invasive assessment of stable chest pain patients, both functional and anatomical information is of interest and may be provided by non-invasive FFR derived from CT (FFRCT). The FORECAST trial (fractional flow reserve derived from computed tomography coronary angiography in the assessment and management of stable chest pain) randomised 1400 patients with stable chest pain to CTCA (including FFRCT for stenoses $>40 \%$ ) vs. usual care [105]. Use of FFRRCT was associated with a reduction in referral for invasive angiography $(19.4 \%$ vs. $25.0 \% ; p=0.01)$ but this did not reduce cost and MACE rates were similar. Thus, the additional benefit of FFRCT in clinical practice remains unclear.

\section{Advances in Atrial Fibrillation and Electrophysiology}

\section{Rhythm Control}

In patients with established AF rhythm vs. rate control has not been shown to be of benefit for hard outcomes [106] but until now the optimal strategy for recent onset AF has been less clear. EAST-AFNET 4 (Early treatment of atrial fibrillation for stroke prevention) randomised 2789 adults with recent ( $<12$ months) AF to routine rhythm control (with anti-arrhythmics and ablation in $8 \%$ ) vs. the initial rate control (and rhythm control only if symptomatic) [107]. The trial was stopped at a median of 5.1 years because of the routine rhythm strategy showing a significant reduction in the primary outcome of $\mathrm{CV}$ events (CV death, stroke, heart failure hospitalisation or ACS) (3.9 vs. 5 per 100 person-years; HR 0.79 [0.66 to 0.94]; $p=0.005$ ). There was no significant difference in the primary safety outcome or time in hospital. This important trial is likely to inform guidelines and shift practice to more intensive rhythm control in patients with recent onset AF.

Current guidelines recommend a trial of one or more antiarrhythmic drugs before consideration of ablation in patients with AF $[108,109]$. The Early Aggressive Invasive Intervention for Atrial Fibrillation (EARLY-AF) trial for rhythm control randomised 303 patients with symptomatic, untreated, paroxysmal AF to ablation using a cryothermy balloon vs. antiarrhythmic drug therapy. All patients received an implantable cardiac monitoring device to detect recurrence of atrial tachyarrhythmia [110]. At 1 year, ablation was associated with significantly less AF recurrence (42.9\% vs. $67.8 \%$; $p<0.001$ ) and less symptomatic arrhythmia (11\% vs. $26.2 \%$; HR $0.3995 \%$ CI [0.22 to 0.68$]$ ) but without excess safety concerns. Further study is required to determine the durability and effect on long-term CV outcomes.

\section{AF Screening}

Given that the first clinical presentation of $\mathrm{AF}$ can be with disabling or fatal stroke, screening for AF may be of clinical value. The mSToPS trial (mHealth screening to prevent strokes) compared 1718 patients given an ECG patch for a median time of 24.7 days (Zio patch, iRhythm Technologies) vs. 3371 observational matched controls [111] (Fig. 6). Patients given a patch

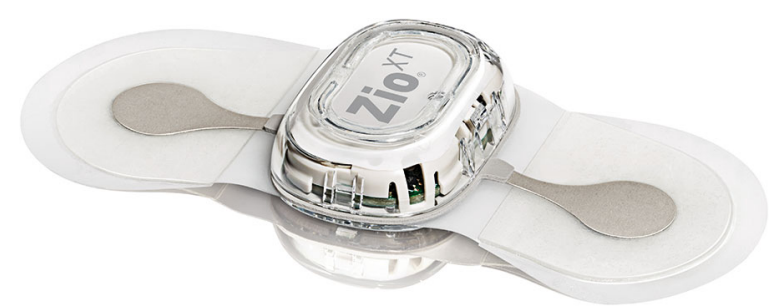

Fig. 6 Xio XT ECG patch (Image supplied courtesy of iRhythm Technologies Ltd.) 
had a higher incidence of new AF diagnosis $(11.4 \%$ vs. $7.7 \% ; p<0.01)$ and a lower incidence of the combined primary endpoint (death, stroke, systemic embolism or MI) at a median of 29 months ( 4.5 vs. 5.5 events per 100 person-years; adjusted HR $0.79 ; p=0.01$ ). In the subgroup diagnosed with $\mathrm{AF}$, the reduction in the primary endpoint reduction was even greater (8.4 vs. 13.8 events per 100 person-years; HR $0.53 ; p<0.01)$. Limitations include use of claims data for endpoints and low overall use of anticoagulants. A larger randomised trial would be of interest to confirm whether screening for $\mathrm{AF}$ with the Zio patch can reduce ischaemic stroke.

The VITAL AF trial randomised patients $>65$ years (mean age 74 years) attending a primary care network to single-lead ECG rhythm screening (AliveCor) $(n=15,397$ patients, 38,891 encounters, 91\% acceptance) vs. usual care $(n=15,325$ patients, 40,459 encounters) [112]. In contrast to mSToPS, AliveCor screening in practice was not associated with a higher rate of new AF diagnosis overall $(1.74 \%$ vs. $1.60 \% ; p=0.33)$, although there was increased detection of new $\mathrm{AF}$ in those $>85$ years (absolute risk increase 1.88\% [95\% CI 0.27 to 3.35]; NNT = 53). This suggests, however, the association between point of care screening and detection of new AF was stronger in patients aged $\geq 85$ with an absolute risk difference $1.88 \%$ and number needed to screen of 53. While VITAL-AF does not support broad population screening in a primary care setting, it suggests a benefit for targeted screening of patients at highest risk.

While the potential for new onset AF during the first few days after cardiac surgery is well recognised, the incidence of new AF during the first few weeks after discharge from cardiac surgery has been poorly defined. SEARCH AF randomised 336 post-cardiac surgery patients with no prior history of AF (but mean CHA2DS2-VASc score of 4) to 30 days of continuous ECG monitoring (using a self-adhesive patch) vs. usual care [113]. Patch monitoring was associated with a significantly higher diagnosis of new $\mathrm{AF} /$ flutter $\geq 6 \mathrm{~min} \quad(19.6 \%$ vs. $1.7 \% ; p<0.001)$. SEARCH-AF was not powered for clinical outcomes but highlights a significant burden of delayed postoperative AF. A larger randomised trial, including $\mathrm{OAC}$ if $\mathrm{AF}$ is detected, would be great interest.

\section{Primary Prevention of AF}

There has been a paucity of randomised trials with respect to AF primary prevention. VITAL Rhythm [114], a substudy of the VITAL trial (which reported on CV events), reported findings from 25,119 patients (mean aged 67 years) randomised in a $2 \times 2$ placebo-controlled design to vitamin D3 (2000 IU/day), omega-3 fatty acids (EPA/DHA mixture $1 \mathrm{~g} /$ day) or both. At 5 years, no significant difference was seen in AF incidence with omega-3 fatty acid vs. placebo (469 vs. 431 , HR $1.09,95 \%$ CI 0.96 to 1.24 , $p=0.19$ ) or vitamin D3 vs. placebo (HR 1.09, $95 \%$ CI 0.96 to $1.25, p=0.19$ ). Whether other primary prevention strategies such as blood pressure or weight reduction or new drugs can successfully reduce AF incidence requires further research.

Education may improve utilisation of OAC for stroke prevention in patients with AF but may be time consuming. The previous IMPACT AF trial reported use of multiple resources including educational brochures, web-based and video educational materials in addition to health care professional interaction was associated with a $9.1 \%$ improvement in adherence to OAC [115]. The IMPACT-AFib trial randomised $47,333 \mathrm{AF}$ patients (not on $\mathrm{OAC}$ but $\mathrm{CHA}_{2} \mathrm{DS}_{2-}$ VASc score $\geq 2$ ) to a single mailed educational intervention to patient and healthcare provider vs. usual care [116]. Receipt of a single mailed intervention was not associated with increased initiation of OAC over the subsequent year (9.89\% vs. 9.80\%; OR $1.01 ; 95 \%$ CI 0.95 to 1.07 ) suggesting that effective patient education may require repeated contacts.

\section{Out of Hospital Arrest}

Survival following out of hospital cardiac arrest remains poor and to date, and no medications have been proven to improve long-term outcomes. In animal models of cardiac arrest, the use of sodium nitrite during resuscitation increased survival by almost $50 \%$. In a prospective clinical trial, 1492 patients with 
out-of-hospital cardiac arrest (22\% ventricular fibrillation) were randomised to low-dose sodium nitrite vs. high-dose sodium nitrite vs. placebo during active resuscitation [117]. In contrast to the pre-clinical data, sodium nitrite was not associated with any difference in the primary endpoint of survival to hospital admission ( $\sim 40 \%$ in each groups; $p>0.5)$, survival in the ventricular fibrillation subgroup or survival to hospital discharge. The poor prognosis associated with out of hospital cardiac arrest remains a critical area for future research.

\section{Advances in Heart Failure}

\section{SGLT2 Inhibitors in Heart Failure}

Sodium-glucose Cotransporter-2 (SGLT2) inhibitors have previously been shown to be beneficial for patients with heart failure (HF) with and without type 2 diabetes (T2D) [2]. Two new studies evaluated sotagliflozin-somewhat different to previous drugs being a dual SGLT inhibitor (inhibiting glucose absorption in the gut via SGLT1 and inhibiting glucose resorption in the kidney via SGLT2).

The SOLOIST-WHF trial (effect of sotagliflozin on $\mathrm{CV}$ events in patients with type 2 diabetes post-worsening heart failure) randomised 1222 patients with T2D and recent HF admission to sotagliflozin vs. placebo [118]. Over an average follow-up of 9 months, sotagliflozin was associated with a $33 \%$ reduction in the primary endpoint (CV death or hospitalisations/urgent visits for HF) (51.0\% vs. $76.34 \%$; HR 0.67; 95\% CI $0.52-0.0 .85$; $p<0.001$ ).

In the SCORED trial (effect of sotagliflozin on $\mathrm{CV}$ and renal events in patients with type 2 diabetes and moderate renal impairment who are at $\mathrm{CV}$ risk), 10,584 patients with T2D and chronic kidney disease (CKD) were randomised to sotagliflozin vs. placebo [119]. Over an average follow-up of 16 months, sotagliflozin was associated with a $26 \%$ reduction in the primary endpoint (CV death or hospitalisations/urgent visits for HF) (5.6 vs. 7.5 events per 100 patientyears; HR 0.74 ; $95 \%$ CI 0.63 to $0.88 ; p<0.001$ ).

Whether the dual action of sotagliflozin is of added clinical benefit to SGLT2 inhibition alone remains uncertain, but the clinical settings were of practical interest in that SOLOIST-WHF supports commencing therapy during an acute admission with HF and SCORED supports initiation in patients with CKD.

Also in the setting of CKD, the DAPA-CKD trial (dapagliflozin and prevention of adverse outcomes in chronic kidney disease) [120] randomised 4304 patients with CKD (eGFR $25-75 \mathrm{ml} / \mathrm{min}$ ) to dapagliflozin vs. placebo. The trial was stopped early because of efficacy at a median of 2.4 years, with dapagliflozin showing a $44 \%$ reduction in the primary endpoint (sustained decline in eGFR $\geq 50 \%$, end-stage kidney disease or renal death) $(9.2 \%$ vs. $14.5 \%$; HR $0.61,95 \%$ CI 0.51 to $0.72 ; p<0.001$ ), a $29 \%$ reduction in $\mathrm{CV}$ death or HF hospitalisation (HR $0.71 ; 95 \%$ CI 0.55 to $0.92 ; p=0.009$ ) and a $31 \%$ reduction in all-cause mortality $(4.7 \%$ vs. $6.8 \%$; HR $0.69 ; 95 \%$ CI 0.53 to $0.88 ; p=0.004)$. This eagerly awaited study confirms that underlying renal dysfunction does not attenuate the CV outcome efficacy of dapagliflozin and that it may have a role in reducing worsening renal function for patients with CKD with and without T2D.

Building further on the case for use of SGLT2 inhibitors as HF medications in their own right, the EMPEROR-reduced trial (Empagliflozin outcome trial in patients with chronic HF with reduced ejection fraction) randomised 3730 patients with $\mathrm{HF}(\mathrm{EF}<40 \%)$ to empagliflozin vs. placebo [121]. At an average of 16 months, empagliflozin showed a $25 \%$ reduction in the primary outcome (CV death or hospitalisation for worsening HF) $(19.4 \%$ vs. $24.7 \%$; HR 0.75 , $95 \%$ CI 0.65 to $0.86 ; p<0.001)$. The benefit was consistent regardless of whether or not the patient had T2D (with diabetes HR 0.72; 95\% CI 0.60 to 0.87 vs. without diabetes HR $0.78 ; 95 \%$ CI 0.64 to 0.97 ).

The mechanisms of benefit of SGLT2 inhibitors in HF remain incompletely understood. The SUGAR-DM-HF trial (StUdies of empaGliflozin and its cArdiovascular, Renal and metabolic effects in patients with Diabetes Mellitus and Heart Failure) randomised 105 patients with $\mathrm{HF}$ and diabetes/pre-diabetes to empagliflozin vs. placebo [122]. CMR performed at baseline and 36 weeks showed empagliflozin 
was associated with a favourable reduction in $\mathrm{LV}$ end-systolic volume (by $6.0 \mathrm{ml} / \mathrm{m}^{2}[-10.8$ to -1.2$] ; p=0.015)$, $\mathrm{LV}$ end diastolic volume (by $8.2 \mathrm{ml} / \mathrm{m}^{2}[-13.7$ to -2.6$] ; p=0.0042$ and NT-proBNP by $28 \% ; p=0.038$, suggesting that reverse $\mathrm{LV}$ remodelling is one mechanism by which SGLT2 inhibitors improve HF outcomes, but further research is needed.

\section{Heart Failure with Reduced Ejection Fraction: Further Strategies}

Omecamtiv mecarbil is a novel, selective cardiac myosin activator that has been shown to improve cardiac function and to decrease ventricular volumes, heart rate and NT-proBNP in patients with chronic HF. The GALACTIC-HF trial (Global approach to lowering adverse cardiac outcomes through improving contractility in heart failure) [123] randomised 8256 patients with $\mathrm{HF}$ and $\mathrm{EF}<35 \%$ to omecamtiv vs. placebo. At a median of 21.8 months, Omecamtiv was associated with an $8 \%$ reduction in the primary outcome (CV death or first HF event) (37\% vs. $39.1 \%$; HR $0.92 ; 95 \%$ CI 0.86 to $0.99 ; p=0.03$ ). Although positive, this magnitude of benefit appears limited compared with other new HF drugs such as sacubitril-valsartan or SGLT2 inhibitors. Of note, as Omecamtiv has no effect on blood pressure it may be useful for patients whose drug titration is limited by hypotension.

Another novel therapy reported on this year was vericiguat, a novel oral soluble guanylate cyclase (sGC) stimulator that acts via the nitric oxide (NO) pathway. NO exerts its beneficial activity via binding to a heme group on sGC but oxidative stress in CV disease may change sGCassociated heme from a ferrous to ferric state, producing a relatively NO-resistant state. Vericiguat, as an sGC stimulator, maintains the enzyme in its active configuration by stabilising its nitrosyl-heme interaction enabling increased cGMP production independent of NO and synergistically with NO. The VICTORIA trial (Vericiguat global study in subjects with heart failure with reduced ejection fraction) randomised 5050 patients with relatively advanced HF (40\% NYHA III-IV) and EF $<45 \%$ to vericiguat vs. placebo [124]. At an average follow-up of 10.8 months, vericiguat was associated with a $10 \%$ reduction in the primary outcome of $\mathrm{CV}$ death or first hospitalisation for HF $(35.5 \%$ vs. $38.5 \%$; HR 0.90; $95 \%$ CI 0.82 to $0.98 ; p=0.02$ ). VICTORIA suggests vericiguat may be of use as additional therapy particularly in those with more advanced HF. As black patients may benefit particularly from NO pathway augmentation but made up only $5 \%$ of the VICTORIA cohort, further research in this patient subgroup may be of value.

The benefit of IV iron in patients with chronic HF and iron deficiency is well established [125] but the effect of IV iron in acute HF has not been studied. The AFFIRM-AHF trial (a study to compare ferric carboxymaltose with placebo in patients with acute heart failure and iron deficiency) randomised 1132 patients hospitalised with acute $\mathrm{HF}, \mathrm{EF}<50 \%$ and iron deficiency to IV ferric carboxymaltose (Ferinject) vs. placebo [126]. At 1 year, IV iron was associated with a strong trend to reduced $\mathrm{CV}$ death or HF hospitalisation (57.2 vs. 72.5 per 100 patient-years; RR 0.79 ; $95 \%$ CI 0.62 to 1.01 ; $p=0.059)$ and driven by a significant reduction in HF hospitalisation (RR 0.74 ; 95\% CI 0.58 to $0.94, p=0.013)$. Thus, AFFIRM-AHF shows that for appropriate patients, it is reasonable to commence IV iron during the acute admission rather than having to wait until a convalescent period (Table 4).

Table 4 Rate of events in the AFFIRM-AHF [126]

\begin{tabular}{lllll}
\hline & $\begin{array}{l}\text { Ferric carboxymaltose } \\
(\boldsymbol{n}=\mathbf{5 5 8})\end{array}$ & $\begin{array}{l}\text { Placebo } \\
(\boldsymbol{n = 5 5 0 )}\end{array}$ & $\begin{array}{l}\text { Hazard ratio (95\% confidence } \\
\text { interval) }\end{array}$ & $\boldsymbol{p}$ value \\
\hline $\begin{array}{l}\text { Heart failure hospitalisations and } \\
\text { CV deaths }\end{array}$ & $293(52.5 \%)$ & $372(67.6 \%)$ & $0.79(0.62-1.01)$ & 0.059 \\
Heart failure hospitalisations & $217(38.9 \%)$ & $294(53.5 \%)$ & $0.74(0.58-0.94)$ & 0.0013 \\
CV deaths & $77(13.8 \%)$ & $78(14.2 \%)$ & $0.96(0.70-1.32)$ & 0.81 \\
\hline
\end{tabular}


Given the increasing complexity of HF drug regimens, methods to educate and involve patients in their care may improve adherence and outcomes. The EPIC-HF study (Electronic health record-leveraged, patient-centered, intensification of chronic care for HF) randomised 306 patients to receive patient-activation tools (video and 1-page checklist delivered electronically 1 week, 3 days and $24 \mathrm{~h}$ prior to a cardiology clinic visit) vs. usual care [127]. By 30 days post clinic those receiving the additional intervention had significantly greater initiation or intensification of guideline directed medical therapy ( $49 \%$ vs. $29.7 \% ; p=0.001)$ supporting the value of this relatively inexpensive but patient-orientated strategy.

\section{Heart Failure with Preserved Ejection Fraction} Finding effective treatment for HF with preserved EF (HFpEF) has been elusive. While sacubitril-valsartan was not found to reduce $\mathrm{CV}$ death or HF hospitalisation, exploratory secondary endpoints, including improvements in NYHA class and quality of life, had hinted at a potential benefit. The PARALLAX study randomised 2566 patients with NYHA III-IV HF and $\mathrm{EF}>40 \%$ to sacubitril-valsartan vs. usual care [128]. The trial met one of its co-primary endpoints in that sacubitril-valsartan was associated with a $16 \%$ greater reduction in NT-proBNP at 12 weeks $(p<0.0001)$ but this was attenuated to non-significance between week 12 and 24 . The second co-primary endpoint, 6-min walk test at 24 weeks, did not show any significant difference between groups.

The concept of optimal blood pressure range in $\mathrm{HF}$ was examined in an interesting new analysis of the PARAGON-HF trial whereby the study cohort were divided into quartiles based on SBP, regardless of treatment arm [129]. The event rate/100 patient years of the primary composite endpoint (CV death or HF hospitalisation) was 15.2 in patients with SBP $<120 \mathrm{mmHg}$ vs. 11.4 events for SBP 120-$129 \mathrm{mmHg}$ vs. 12.2 for SBP $130-139 \mathrm{mmHg}$ and 15.6 for SBP $>140 \mathrm{mmHg}$. These results support a relationship between SBP and HFpEF events although it is unclear whether SBP is a prognostic surrogate or should be seen as a primary therapeutic target.

\section{Hypertrophic Cardiomyopathy}

Only a few therapeutic options are available for patients with hypertrophic cardiomyopathy (HCM) with significant obstruction and they are often poorly tolerated. The EXPLORER-HCM trial randomised 251 patients with $\mathrm{HCM}$ and a left ventricular outflow tract (LVOT) gradient 

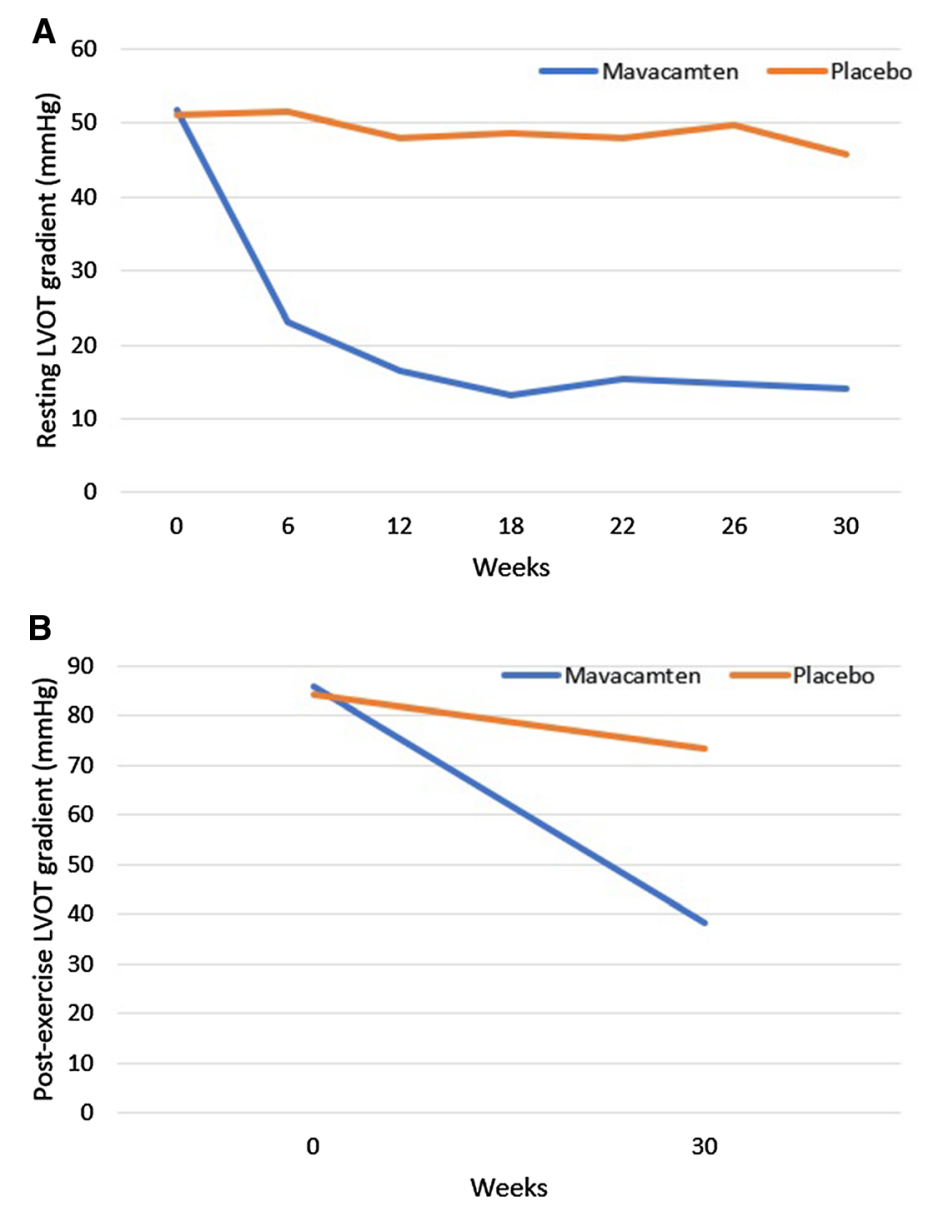

Fig. 7 Changes in resting (a) and post-exercise (b) LVOT gradients in patients taking Mavacamten compared with placebo [130]

of $>50 \mathrm{mmHg}$ to mavacamten (a cardiac myosin inhibitor) vs. placebo [130] (Fig. 7). The primary endpoint was $\geq 1.5 \mathrm{ml} / \mathrm{kg} / \mathrm{min}$ increase in peak oxygen consumption (pVO2) plus $\geq 1$ class improvement in NYHA or $\geq 3.0$ $\mathrm{ml} / \mathrm{kg} / \mathrm{min} \mathrm{pVO} 2$ increase without deterioration in NYHA class. At 30 weeks, mavacamten was associated with greater likelihood of achieving the primary endpoint ( $37 \%$ vs. $17 \% ; p=0.0005$ ) and greater reduction in the post-exercise LVOT gradient $\quad(-47 \mathrm{mmHg} \quad$ vs. $-10 \mathrm{mmHg}$; $p<0.0001$ ), and $27 \%$ achieved LVOT gradient $<30 \mathrm{mmHg}$ with absence of symptoms. The functional results of this study are very encouraging and suggest that mavacamten may even enable some patients to avoid myectomy/ septal ablation intervention although further studies would be required to confirm this.

\section{Pericardial Disease}

Up to $30 \%$ of patients presenting with a pericarditis may have a recurrent episode despite treatment with colchicine leading to significant morbidity and use of healthcare resources. Rilonacept, an interleukin- $1 \alpha$ and interleukin$1 \beta$ cytokine trap, given by subcutaneous injection, was previously shown to reduce pericardial inflammation [131]. In the RHAPSODY trial 86 patients with recurrent pericarditis and elevated C-reactive protein level despite standard treatment were initiated on rilonacept for a 12 -week run-in (with pain resolution by a median of 5 days and C-reactive protein normalisation by a median of 7 days). Following the run-in, the 61 patients who showed clinical response were randomised to ongoing rilonacept vs. placebo [132]. By 8.6 weeks, ongoing rilonacept was 
associated with a marked reduction in recurrence of pericarditis (7\% vs. 74\%; HR 0.04; 95\% CI 0.01 to $0.18 ; p<0.001)$ suggesting this novel drug may become an important therapeutic option for patients with recurrent pericarditis.

\section{LIMITATIONS}

While all summarised trials have been presented at major cardiology conferences in 2020, not all trials have been published in peer-reviewed journals.

\section{CONCLUSION}

This article has highlighted and summarised the key trials that were published and presented in the field of cardiology during 2020. Many of these studies will help guide clinical practice and influence guideline development. Others have shown encouraging early data to guide future therapeutic strategies.

\section{ACKNOWLEDGEMENTS}

Funding. No funding or sponsorship was received for this study or publication of this article.

Authorship. All named authors meet the International Committee of Medical Journal Editors (ICMJE) criteria for authorship for this manuscript, take responsibility for the integrity of the work as a whole, and have given final approval for the version to be published.

Disclosures. Aileen Kearney, Katie Linden and Patrick Savage have nothing to disclose. Ian Menown has received grants to institution, honoraria and/or conference sponsorship from Biosensors, Meril Life, Orbus Neich, Bayer, Boehringer Ingelheim, and Daichii Sankyo. Ian Menown is a member of the journal's editorial board.
Compliance with Ethics Guidelines. This article is based on previously conducted studies and does not involve any new studies of human or animal subjects performed by any of the authors.

Data Availability. Data sharing is not applicable to this article as no datasets were generated or analyzed during the current study.

Open Access. This article is licensed under a Creative Commons Attribution-NonCommercial 4.0 International License, which permits any non-commercial use, sharing, adaptation, distribution and reproduction in any medium or format, as long as you give appropriate credit to the original author(s) and the source, provide a link to the Creative Commons licence, and indicate if changes were made. The images or other third party material in this article are included in the article's Creative Commons licence, unless indicated otherwise in a credit line to the material. If material is not included in the article's Creative Commons licence and your intended use is not permitted by statutory regulation or exceeds the permitted use, you will need to obtain permission directly from the copyright holder. To view a copy of this licence, visit http://creativecommons.org/licenses/by$\mathrm{nc} / 4.0 /$.

\section{REFERENCES}

1. Henry TD. Initial outcomes from NACMI the North American COVID-19 STEMI registry. Presented at Transcatheter cardiovascular therapeutics virtual meeting (TCT Connect), 14 Oct 2020.

2. Linden K, Mailey J, Kearney A, Menown IBA, et al. Advances in clinical cardiology 2019: a summary of key clinical trials. Adv Ther. 2020;37:2620-45.

3. Bangalore S, Maron DJ, Stone GW, Hochman JS. Routine revascularization versus initial medical therapy for stable ischemic heart disease: a systematic review and meta-analysis of randomized trials. Circulation. 2020;142(9):841-57.

4. Outcomes with intermediate left main disease on coronary CT angiography in the ISCHEMIA trial (ISCHEMIA Intermediate LM Substudy). In: SCAI 
2020 scientific sessions virtual conference, 14-16 May 2020.

5. Joy G, Eissa H. Do EXCEL and NOBLE translate into real world? A 5-year observational study of left main stem outcomes. Open Heart. 2020;7(2):e001347.

6. Park DW, Ahn JM, Park H, et al. Ten-year outcomes after drug-eluting stents versus coronary artery bypass grafting for left main coronary disease: extended follow-up of the PRECOMBAT trial. Circulation. 2020;141(18):1437-46.

7. Galassi AR, Tomasello SD, Reifart N, Werner GS, Sianos G. In-hospital outcomes of percutaneous coronary intervention in patients with chronic total occlusion: insights from the ERCTO (European Registry of Chronic Total Occlusion) registry. EuroIntervention. 2011;7(4):472-9.

8. $\mathrm{Xu} \mathrm{B}$, et al. Coronary artery bypass grafting and percutaneous coronary intervention in patients with chronic total occlusion and multivessel disease. In: EuroPCR virtual sessions, 18-21 May 2020.

9. Gaudino M, Benedetto U, Fremes S, et al. Association of radial artery graft vs saphenous vein graft with long-term cardiovascular outcomes among patients undergoing coronary artery bypass grafting: a systematic review and meta-analysis. JAMA. 2020;324(2):179-87.

10. Steg P, et al. REALITY: a trial of transfusion strategies for myocardial infarction and anemia. In: Virtual presentation; European Society of Cardiology Virtual Congress, 1 Sept 2020.

11. Zhang JJ, Ye F, Xu K, et al. Multicentre, randomized comparison of two-stent and provisional stenting techniques in patients with complex coronary bifurcation lesions: the DEFINITION II trial. Eur Heart J. 2020;41(27):2523-36.

12. Stone GW, Kimura T, Gao R, et al. Time-varying outcomes with the absorb bioresorbable vascular scaffold during 5-year follow-up: a systematic metaanalysis and individual patient data pooled study. JAMA Cardiol. 2019;4(12):1261-9. https://doi.org/ 10.1001/jamacardio.2019.4101.

13. Verheye S, Vrolix M, Montorfano M, et al. Twelvemonth clinical and imaging outcomes of the uncaging DynamX Bioadaptor system. EuroIntervention. 2020;16(12):e974-81.

14. Menown IBA, et al. Cobalt chromium biolimuseluting stents compared with stainless steel biolimus-eluting stents: final results of the BMX alpha registry. Presented at TCT 2020.

15. Kandzari DE, Koolen JJ, Doros G, et al. Ultrathin bioresorbable-polymer sirolimus-eluting stents versus thin durable-polymer everolimus-eluting stents for coronary revascularization: 3-year outcomes from the randomized BIOFLOW $\mathrm{V}$ trial. JACC Cardiovasc Interv. 2020;13(11):1343-53.

16. Kereiakes DJ, et al. OPTIMIZE IDE: trial prospective, randomized evaluation of sirolimus-eluting coronary stents with fixed-wire and rapid-exchange delivery systems and a novel bioresorbable drug carrier. Presentation at TCT connect, the 32nd annual transcatheter cardiovascular therapeutics scientific symposium of the cardiovascular research foundation held online, 14-18 Oct 2020.

17. Lansky A. Novel healing-targeted DES with synchronized antiproliferative drug delivery to target smooth muscle cell proliferation after DES implantation in coronary artery disease. Primary results of the PIONEER III Trial. Presented at AHA 2020, 15 Nov 2020.

18. Byrne $\mathrm{R}$, et al. COBRA PzF stenting to REDUCE duration of triple therapy. Presented at Transcatheter cardiovascular therapeutics virtual meeting (TCT connect), 17 Oct 2020.

19. Ferrari R, Ford I, Fox K, Challeton JP, Correges A, Tendera M, Widimský P, Danchin N, ATPCI investigators. Efficacy and safety of trimetazidine after percutaneous coronary intervention (ATPCI): a randomised, double-blind, placebo-controlled trial. Lancet. 2020;396(10254):830-8.

20. van de Hoef TP, Echavarria-Pinto M, Meuwissen M, Stegehuis VE, Escaned J, Piek JJ. Contribution of age-related microvascular dysfunction to abnormal coronary: hemodynamics in patients with ischemic heart disease. JACC Cardiovasc Interv. 2020;13(1): 20-9.

21. Escaned J. Two-year outcomes of patients with revascularization deferral based on FFR or iFR measurements: a pooled, patient level analysis of DEFINE FLAIR and iFR SWEDEHEART trials. Presented on: 25 June 2020. PCR e-Course 2020.

22. Waksman R, Legutko J, Singh J, et al. FIRST: fractional flow reserve and intravascular ultrasound relationship study. J Am Coll Cardiol. 2013;61(9): 917-23.

23. Vergallo E, et al. Optical coherence tomography measures predicting fractional flow reserve: the OMEF study. Presented at SCAI May 2020.

24. Johnson N. DEFINE-FLOW: an observational study of deferred lesions after FFR and CFR assessment. Presented at Transcatheter cardiovascular therapeutics virtual meeting (TCT connect), 16 Oct 2020. 
25. Kibel A, Selthofer-Relatic K, Drenjancevic I, et al. Coronary microvascular dysfunction in diabetes mellitus. J Int Med Res. 2017;45(6):1901-29.

26. Kedhi E. Combined optical coherence tomography and fractional flow reserve assessment to better predict adverse event outcomes in DM patients. Presented at: TCT 2020, 14 Oct 2020.

27. Collison D. TARGET FFR, physiology-guided optimization of PCI, a randomized controlled trial. Presented at Transcatheter cardiovascular therapeutics virtual meeting (TCT connect), 16 Oct 2020.

28. Erlinge D, for the PROSPECT II investigators. PROSPECT II: a prospective natural history study using NIRS-IVUS imaging in patients with acute myocardial infarction. Presented at Transcatheter cardiovascular therapeutics virtual meeting (TCT connect), 14 Oct 2020 .

29. Stone GW, Maehara A, Ali ZA, et al. Percutaneous coronary intervention for vulnerable coronary atherosclerotic plaque. J Am Coll Cardiol. 2020;76(20):2289-301.

30. Daida H, Dohi T, Fukushima Y, Ohmura H, Miyauchi K. The goal of achieving atherosclerotic plaque regression with lipid-lowering therapy: insights from IVUS trials. J Atheroscler Thromb. 2019;26(7):592-600.

31. Gao XF, Ge Z, Kong XQ, et al. Three-year outcomes of the ULTIMATE trial comparing intravascular ultrasound versus angiography-guided drug-eluting stent implantation. JACC Cardiovasc Interv. 2020. (Epub ahead of print).

32. Toff WD. The United Kingdom transcatheter aortic valve implantation (UK TAVI) trial. Presented at ACC/WCC, 28-30 Mar 2020.

33. Mack MJ, Leon MB, Thourani VH, et al. Transcatheter aortic-valve replacement with a balloonexpandable valve in low-risk patients. N Engl J Med. 2019;380(18):1695-705.

34. Mack MJ. Two-year clinical and echocardiographic outcomes from the PARTNER 3 low-risk randomized trial. Presented at ACC/WCC, 28-30 Mar 2020.

35. Forrest JK, Ramlawi B, Deeb GM, et al. Transcatheter aortic valve replacement in low-risk patients with bicuspid aortic valve stenosis. JAMA Cardiol. 2020. (Epub ahead of print).

36. Graziani F, Mencarelli E, Burzotta F, et al. Early hemodynamic and structural impact of transcatheter aortic valve replacement in pure aortic regurgitation. J Am Coll Cardiol Cardiovasc Interv. 2020;13(21):2582-4.
37. Lanz J, Kim WK, Walther T, et al. Safety and efficacy of a self-expanding versus a balloon-expandable bioprosthesis for transcatheter aortic valve replacement in patients with symptomatic severe aortic stenosis: a randomised non-inferiority trial. Lancet. 2019;394:1619-28.

38. Walther T. SCOPE I: One-year outcomes of a randomized trial comparing a self -expanding to a balloon-expandable transcatheter aortic valve. Presented at Transcatheter cardiovascular therapeutics virtual meeting (TCT connect), 17 Oct 2020.

39. Tamburino C, Bleiziffer S, Thiele H, et al. Comparison of self-expanding bioprostheses for transcatheter aortic valve replacement in patients with symptomatic severe aortic stenosis: the SCOPE 2 randomized clinical trial. Circulation, 2020, (Epub ahead of print).

40. Baumgartner H, Falk V, Bax JJ, et al. 2017 ESC/ EACTS Guidelines for the management of valvular heart disease The Task Force for the Management of Valvular Heart Disease of the European Society of Cardiology (ESC) and the European Association for Cardio-Thoracic Surgery (EACTS). Eur Heart J. 2017;38(36):2739.

41. Brouwer J, Nijenhuis VJ, Delewi R, et al. Aspirin with or without clopidogrel after transcatheter aortic-valve implantation. N Engl J Med. 2020;383: 144-57.

42. Rodés-Cabau J, Masson J-B, Welsh RC, et al. Aspirin versus aspirin plus clopidogrel as antithrombotic treatment following transcatheter aortic valve replacement with a balloon-expandable valve: the ARTE (aspirin versus aspirin + clopidogrel following transcatheter aortic valve implantation) randomized clinical trial. JACC Cardiovasc Interv. 2017;10: 1357-65.

43. Nijenhuis VJ, Brouwer J, Delewi R, et al. Anticoagulation with or without clopidogrel after transcatheter aortic-valve implantation. N Engl J Med. 2020;382:1696-707.

44. Moses JW. A randomized evaluation of the TriGUARD3 ${ }^{\mathrm{TM}}$ cerebral embolic protection device to reduce the impact of cerebral embolic lesions after transcatheter aortic valve implantation the REFLECT II trial. Presented at the Transcatheter cardiovascular therapeutics virtual meeting (TCT connect), 15 Oct 2020.

45. Webb JG, Hensey M, Szerlip M, et al. 1-Year outcomes for transcatheter repair in patients with mitral regurgitation from the CLASP study. J Am Coll Cardiol Cardiovasc Interv. 2020;13(20): 2344-57. 
46. Edwards PASCAL CLASP IID/IIF Pivotal Clinical Trial (CLASP IID/IIF) (ClinicalTrials.gov website). https://www.clinicaltrials.gov/ct2/show/ NCT03706833. Accessed 5 June 2020.

47. Kar S. One-year outcomes with third generation MitraClip NTR and XTR systems. Presented at: TCT 2020, 16 Oct 2020.

48. Qasim A, Kar S, Weissman N, et al. Everest II REALISM: early success rates of MitraClip placement by etiology of regurgitation and mitral valve morphology. Presented at: American College Cardiology 2016 Scientific Sessions, Chicago, IL, 4 Apr 2016.

49. Godino C, Munafo A, Scotti A, et al. MitraClip in secondary mitral regurgitation as a bridge to heart transplantation: 1-year outcomes from the International MitraBridge Registry. J Heart Lung Transplant. 2020;39:1353-62.

50. Lurz P, Unterhuber M, Rommel KP, et al. Closure of iatrogenic atrial septal defect following transcatheter mitral valve repair: the randomized MITHRAS trial. Circulation. 2021;143(3):292-4.

51. Lurz P. Percutaneous edge-to-edge repair for tricuspid regurgitation: 1-year outcomes from TRILUMINATE trial. Presented at EuroPCR 2020, 25 June 2020

52. Schwartz GG, Steg PG, Szarek M, et al. Alirocumab and cardiovascular outcomes after acute coronary syndrome. N Engl J Med. 2018;379:2097-107.

53. Jukema JW, Szarek M, Zijlstra LE, et al. Alirocumab in patients with polyvascular disease and recent acute coronary syndrome: ODYSSEY OUTCOMES trial. J Am Coll Cardiol. 2019;74(9):1167-76.

54. Ridker PM, Revkin J, Amarenco P, et al. Cardiovascular efficacy and safety of bococizumab in highrisk patients. N Engl J Med. 2017;376:1527-39.

55. Sabatine MS, Giugliano RP, Keech AC, et al. Evolocumab and clinical outcomes in patients with cardiovascular disease. N Engl J Med. 2017;376: 1713-22.

56. Blom DJ, Harada-Shiba M, Rubba P, et al. Efficacy and safety of alirocumab in adults with homozygous familial hypercholesterolemia: the ODYSSEY HoFH trial. J Am Coll Cardiol. 2020;76(2):131-42.

57. Koskinas KC, Windecker S, Pedrazzini G, et al. Evolocumab for early reduction of LDL cholesterol levels in patients with acute coronary syndromes (EVOPACS). J Am Coll Cardiol. 2019;74(20): 2452-62.
58. Okada T, Doi M, Miyoshi T, et al. Early initiation of evolocumab markedly reduces low-density lipoprotein cholesterol levels after acute myocardial infarction. J Am Coll Cardiol Cardiovasc Interv. E-publication 25 Sept 2020.

59. Rosenson RS, Burgess LJ, Ebenbichler CF, et al. Evinacumab in patients with refractory hypercholesterolemia. N Engl J Med. 2020;383(24):2307-19.

60. Wood FA, Howard JP, Finegold JA, et al. N-of-1 trial of a statin, placebo, or no treatment to assess side effects. N Engl J Med. 2020;383(22):2182-4.

61. Bhatt DL, Steg PG, Miller M, et al. Cardiovascular risk reduction with icosapent ethyl for hypertriglyceridemia. N Engl J Med. 2019;380:11-22.

62. Bhatt DL. EPA levels and cardiovascular outcomes in the reduction of cardiovascular events with icosapent ethyl-intervention trial. Presented at American College of Cardiology annual scientific sessions 2020, Chicago (virtual), 30 Mar 2020.

63. Nicholls SJ, Lincoff AM, Garcia M, et al. Effect of high-dose omega-3 fatty acids vs corn oil on major adverse cardiovascular events in patients at high cardiovascular risk: the STRENGTH randomized clinical trial. JAMA. 2020;324(22):2268-80.

64. Kalstad AA, Myhre PL, Laake K, et al. Effects of n-3 fatty acid supplements in elderly patients after myocardial infarction: a randomized controlled trial. Circulation. E-publication on 15 Nov 2020.

65. Linden K, McQuillan C, Brennan P, Menown IBA. Advances in clinical cardiology 2018: a summary of key clinical trials. Adv Ther. 2019;36(7):1549-73.

66. Mahfoud F, Mancia G, Schmieder R, et al. Renal denervation in high-risk patients with hypertension. J Am Coll Cardiol. 2020;75(23):2879-88.

67. Böhm M, Kario K, Kandzari DE, et al. Efficacy of catheter-based renal denervation in the absence of antihypertensive medications (SPYRAL HTN-OFF MED Pivotal): a multicentre, randomised, shamcontrolled trial. Lancet. 2020;395(10234):1444-51.

68. Rahimi K. Pharmacological blood pressure-lowering for primary and secondary prevention of cardiovascular disease across different levels of blood pressure: an individual participant meta-analysis of 48 randomized clinical trials and 348,854 participants. Presented at the European Society of Cardiology Congress 2020, Virtual, 31 Aug 2020.

69. Tardif JC, Kouz S, Waters DD, et al. Efficacy and safety of low-dose colchicine after myocardial infarction. N Engl J Med. 2019;381:2497-505. 
70. Nidorf SM, Fiolet ATL, Mosterd A, et al. Colchicine in patients with chronic coronary disease. $\mathrm{N}$ Engl J Med. 2020;383(19):1838-47.

71. Yusuf S, Joseph P, Dans A, et al. Polypill with or without aspirin in persons without cardiovascular disease. N Engl J Med. 2021;384(3):216-28.

72. Gaziano JM, Brotons C, Coppolecchia R, et al. Use of aspirin to reduce risk of initial vascular events in patients at moderate risk of cardiovascular disease (ARRIVE): a randomised, double-blind, placebocontrolled trial. Lancet. 2018;392(10152):1036-46.

73. Eisenberg MJ, Hébert-Losier A, Windle SB, et al. Effect of e-cigarettes plus counseling vs counseling alone on smoking cessation a randomized clinical trial. JAMA. 2020;324(18):1844-54.

74. Holt A. Effect of beta blocker therapy following myocardial infarction in optimally treated patients in the reperfusion era - a Danish, nationwide, and registry-based cohort study. Presented at the European Society of Cardiology Congress 2020, virtual, 29 Aug 2020.

75. Lopes RD. Continuing versus suspending angiotensin-converting enzyme inhibitors and angiotensin receptor blockers: impact on adverse outcomes in hospitalized patients with severe acute respiratory distress syndrome coronavirus 2 (SARSCoV-2). Presented at the European Society of Cardiology Congress 2020, virtual, 1 Sept 2020.

76. Jones DW, Minhas S, Fierro JJ, et al. From WOEST to AUGUSTUS: a review of safety and efficacy of triple versus dual antithrombotic regimens in patients with atrial fibrillation requiring percutaneous coronary intervention for acute coronary syndrome. Ann Transl Med. 2019;7(17):405.

77. Alexander JH, Wojdyla D, Vora AN, Thomas L, Granger CB, Goodman SG, Aronson R, Windecker S, Mehran R, Lopes RD. Risk/benefit tradeoff of antithrombotic therapy in patients with atrial fibrillation early and late after an acute coronary syndrome or percutaneous coronary intervention: insights from AUGUSTUS. Circulation. 2020;141(20):1618-27.

78. Pereira NL, Farkouh ME, So D, et al. Effect of genotype-guided oral P2Y12 inhibitor selection vs conventional clopidogrel therapy on ischemic outcomes after percutaneous coronary intervention: the TAILOR-PCI randomized clinical trial. JAMA. 2020;324(8):761-71.

79. Kim BK, Hong SJ, Cho YH, et al. Effect of ticagrelor monotherapy vs ticagrelor with aspirin on major bleeding and cardiovascular events in patients with acute coronary syndrome: the TICO randomized clinical trial. JAMA. 2020;323(23):2407-16.
80. Vranckx P, Valgimigli M, Jüni $\mathrm{P}$, et al. Ticagrelor plus aspirin for 1 month, followed by ticagrelor monotherapy for 23 months vs aspirin plus clopidogrel or ticagrelor for 12 months, followed by aspirin monotherapy for 12 months after implantation of a drug-eluting stent: a multicentre, openlabel, randomised superiority trial. Lancet. 2018;392:940-9.

81. Watanabe H, Domei T, Morimoto T, et al. Effect of 1-month dual antiplatelet therapy followed by clopidogrel vs 12-month dual antiplatelet therapy on cardiovascular and bleeding events in patients receiving PCI: the STOPDAPT-2 randomized clinical trial. JAMA. 2019;321:2414-27.

82. Hahn JY, Song YB, Oh JH, et al. Effect of P2Y12 inhibitor monotherapy vs dual antiplatelet therapy on cardiovascular events in patients undergoing percutaneous coronary intervention: the SMARTCHOICE randomized clinical trial. JAMA. 2019;321: 2428-37.

83. Mehran R, Baber U, Sharma SK, et al. Ticagrelor with or without aspirin in high-risk patients after PCI. N Engl J Med. 2019;381:2032-42.

84. Angiolillo DJ, Baber U, Sartori S, et al. Ticagrelor with or without aspirin in high-risk patients with diabetes mellitus undergoing percutaneous coronary intervention. J Am Coll Cardiol. 2020;75(19): 2403-13.

85. Dangas G, Baber U, Sharma S, et al. Ticagrelor with aspirin or alone after complex PCI. J Am Coll Cardiol. 2020;75(19):2414-24.

86. Kim HS, Kang J, Hwang D, Han JK, Yang HM, Kang HJ, Koo BK, Rhew JY, Chun KJ, Lim YH, Bong JM, Bae JW, Lee BK, Park KW. Prasugrel-based de-escalation of dual antiplatelet therapy after percutaneous coronary intervention in patients with acute coronary syndrome (HOST-REDUCE-POLYTECHACS): an open-label, multicentre, non-inferiority randomised trial. Lancet. 2020;396(10257): 1079-89.

87. Cannon CP, Harrington RA, James S, et al. Comparison of ticagrelor with clopidogrel in patients with a planned invasive strategy for acute coronary syndromes (PLATO): a randomised double-blind study. Lancet. 2010;375:283-93.

88. Mehilli J, Baquet M, Hochholzer W, et al. Randomized comparison of intensified and standard P2Y12-receptor-inhibition before elective percutaneous coronary intervention: the SASSICAIA trial. Circ Cardiovasc Interv. 2020;13:e008649.

89. Silvain J, Lattuca B, Beygui F, et al. Ticagrelor versus clopidogrel in elective percutaneous coronary intervention (ALPHEUS): a randomised, open-label, 
phase 3b trial. Lancet. 2020;S0140-6736(20): 32236-44.

90. McCune C, McKavanagh P, Menown IB. A review of current diagnosis, investigation, and management of acute coronary syndromes in elderly patients. Cardiol Ther. 2015;4(2):95-116.

91. Szummer K, Montez-Rath ME, Alfredsson J, et al. Comparison between ticagrelor and clopidogrel in elderly patients with an acute coronary syndrome. Circulation. 2020;142:1700-8.

92. Tarantini G, Mojoli M, Varbella F, et al. Timing of oral P2Y12 inhibitor administration in patients with non-ST-segment elevation acute coronary syndrome. J Am Coll Cardiol. 2020;76(21):2450-9.

93. Rollini F, Franchi F, Hu J, et al. Crushed prasugrel tablets in patients with STEMI undergoing primary percutaneous coronary intervention: the CRUSH study. J Am Coll Cardiol. 2016;67(17):1994-2004.

94. Parodi G, Xanthopoulou I, Bellandi B, et al. Ticagrelor crushed tablets administration in STEMI patients: the MOJITO study. J Am Coll Cardiol. 2015;65(5):511-2.

95. Vlachojannis G, Wilschut JM, Vogel R, et al. Effect of pre-hospital crushed prasugrel tablets in patients with STEMI planned for primary percutaneous coronary intervention: the randomized COMPARE CRUSH trial. Circulation. 2020;142(24):2316-28.

96. Farag M, Spinthakis N, Srinivasan M, Gorog DA. Should STEMI patients receive opiate analgesia? The morphine paradox. Curr Vasc Pharmacol. 2018;16(5):477-83.

97. Tavenier AH, Hermanides RS, Ottervanger JP, et al. Impact of opioids on P2Y12-receptor inhibition in patients with ST-elevation myocardial infarction who are pre-treated with crushed ticagrelor: opioids and crushed ticagrelor in myocardial infarction evaluation (ON-TIME 3) trial. Eur Heart J Cardiovasc Pharmacother. 2020. https://doi.org/10.1093/ ehjcvp/pvaa095 (Epub ahead of print. PMID: 32730628).

98. Connolly SJ, Ezekowitz MD, Yusuf S, et al. Dabigatran versus warfarin in patients with atrial fibrillation. N Engl J Med. 2009;361(12):1139-51.

99. Granger CB, Alexander JH, McMurray JJ, et al. Apixaban versus warfarin in patients with atrial fibrillation. N Engl J Med. 2011;365(11):981-92.

100. Guimarães HP, Lopes RD, de Barros E Silva PGM, et al. Rivaroxaban in patients with atrial fibrillation and a bioprosthetic mitral valve. $\mathrm{N}$ Engl J Med. 2020;383(22):2117-26.
101. Tamis-Holland JE, Jneid H, Reynolds HR, et al. Contemporary diagnosis and management of patients with myocardial infarction in the absence of obstructive coronary artery disease: a scientific statement from the American Heart Association. Circulation. 2019;139:e891-908.

102. Reynolds HR, Maehara A, Kwong RY, et al. Coronary optical coherence tomography and cardiac magnetic resonance imaging to determine underlying causes of MINOCA in women. Circulation. 2020. https://doi.org/10.1161/CIRCULATIONAHA.120. 052008 (Epub ahead of print. PMID: 33191769).

103. Samad Z, Hakeem A, Mahmood SS, et al. A metaanalysis and systematic review of computed tomography angiography as a diagnostic triage tool for patients with chest pain presenting to the emergency department. J Nucl Cardiol. 2012;19: 364-76.

104. Gray A. Early coronary CT angiography in patients with suspected or provisionally diagnosed acute coronary syndrome: the RAPID CTCA trial. Presented at: AHA 2020, 16 Nov 2020.

105. Curzen N, on behalf of the FORECAST investigators. Fractional flow reserve derived from computed tomography coronary angiography in the assessment and management of stable chest pain. Presented at: TCT 2020, 16 Oct 2020

106. Van Gelder IC, Hagens VE, Bosker HA, et al. A comparison of rate control and rhythm control in patients with recurrent persistent atrial fibrillation. N Engl J Med. 2002;347:1834-40.

107. Kirchhof P, Camm AJ, Goette A, et al. Early rhythmcontrol therapy in patients with atrial fibrillation. N Engl J Med. 2020;383:1305-16.

108. Hindricks G, Potpara T, Dagres N, et al. 2020 ESC guidelines for the diagnosis and management of atrial fibrillation developed in collaboration with the European Association of Cardio-Thoracic Surgery (EACTS). Eur Heart J. 2020. (Epub ahead of print).

109. January CT, Wann LS, Calkins H, et al. 2019 AHA/ ACC/HRS focused update of the 2014 AHA/ACC/ HRS guideline for the management of patients with atrial fibrillation: a report of the American College of Cardiology/American Heart Association Task Force on Clinical Practice Guidelines and the Heart Rhythm Society in collaboration with the Society of Thoracic Surgeons. Circulation. 2019;140(2): e125-51.

110. Andrade JG, Wells GA, Deyell MW, et al. Cryoablation or drug therapy for initial treatment of atrial fibrillation. N Engl J Med. 2020. https://doi.org/10. 
1056/NEJMoa2029980 (Epub ahead of print. PMID: 33197159).

111. Steinhubl SR, et al. 3-year clinical outcomes in a nationwide pragmatic clinical trial of atrial fibrillation screening: mHealth screening to prevent strokes (mSToPS). Presented at AHA 2020.

112. Lubitz SA. Screening for atrial fibrillation in older adults at primary care visits using single lead electrocardiograms: the VITAL-AF trial. Presented at AHA 2020.

113. Verma S, on behalf of the SEARCH-AF investigators. Enhanced monitoring for atrial fibrillation following cardiac surgery: primary results of the SEARCHAF CardioLink trial. Presented at: AHA 2020, 16 Nov 2020.

114. Albert C. The VITAL Rhythm trial: omega-3 fatty acid and vitamin $\mathrm{D}$ supplementation in the primary prevention of atrial fibrillation. Presented at: AHA 2020, 14 Nov 2020.

115. Pokorney S. IMPACT-Afib implementation of stroke prevention in atrial fibrillation. Presented at ESC 2020, 1 Sept 2020.

116. Vinereanu D, Lopes RD, Bahit M, et al. A multifaceted intervention to improve treatment with oral anticoagulants in atrial fibrillation: an international, cluster randomised trial-IMPACT AF. Lancet. $2017 ; 390(10104): 1737-46$.

117. Kim F, et al. Randomized clinical trial of pre-hospital sodium nitrite in out-of-hospital cardiac arrest patients. Presented at ACC/WCC 28-30 Mar 2020.

118. Bhatt DL, Szarek M, Steg PG, et al. Sotagliflozin in patients with diabetes and recent worsening heart failure. N Engl J Med. 2020. https://doi.org/10. 1056/NEJMoa2030183 (Epub ahead of print. PMID: 33200892).

119. Bhatt DL, Szarek M, Pitt B, et al. Sotagliflozin in patients with diabetes and chronic kidney disease. N Engl J Med. 2020. https://doi.org/10.1056/ NEJMoa2030186 (Epub ahead of print. PMID: 33200891).

120. Heerspink HJL, Stefánsson BV, Correa-Rotter R, et al. Dapagliflozin in patients with chronic kidney disease. N Engl J Med. 2020;383(15):1436-46.

121. Packer M, Anker SD, Butler J, et al. Cardiovascular and renal outcomes with empagliflozin in heart failure. N Engl J Med. 2020;383(15):1413-24.

122. Lee MMY, Brooksbank KJM, Wetherall K, et al. Effect of empagliflozin on left ventricular volumes in patients with type 2 diabetes, or prediabetes, and heart failure with reduced ejection fraction (SUGAR-DM-HF). Circulation. 2020. https://doi. org/10.1161/CIRCULATIONAHA.120.052186

(Epub ahead of print. PMID: 33186500).

123. Teerlink JR, Diaz R, Felker GM, et al. Cardiac myosin activation with omecamtiv mecarbil in systolic heart failure. N Engl J Med. 2020. https://doi.org/ 10.1056/NEJMoa2025797 (Epub ahead of print. PMID: 33185990).

124. Armstrong PW, Pieske B, Anstrom KJ, et al. Vericiguat in patients with heart failure and reduced ejection fraction. N Engl J Med. 2020;382(20): 1883-93.

125. Kang CK, Pope M, Lang CC, Kalra PR. Iron deficiency in heart failure: efficacy and safety of intravenous iron therapy. Cardiovasc Ther. 2017;35: e12301.

126. Ponikowski P, Kirwan BA, Anker SD, et al. Ferric carboxymaltose for iron deficiency at discharge after acute heart failure: a multicentre, doubleblind, randomised, controlled trial. Lancet. 2020;396(10266):1895-904.

127. Allen LA, Venechuk G, McIvennan CK, et al. An electronically delivered, patient-activation tool for intensification of medications for chronic heart failure with reduced ejection fraction: the EPIC-HF trial. Circulation. E-publication 17 Nov 2020.

128. Pieske B. Angiotensin receptor neprilysin inhibition compared with individualized medical therapy for comorbidities in patients with heart failure and preserved ejection fraction: the PARALLAX trial. Presented at European Society of Cardiology Congress 2020, virtual, 30 Aug 2020.

129. Selvaraj S, Claggett BL, Böhm M, et al. Systolic blood pressure in heart failure with preserved ejection fraction treated with sacubitril/valsartan. J Am Coll Cardiol. 2020;75(14):1644-56.

130. Olivotto I, Oreziak A, Barriales-Villa R, et al. Mavacamten for treatment of symptomatic obstructive hypertrophic cardiomyopathy (EXPLORER-HCM): a randomised, double-blind, placebo-controlled, phase 3 trial. Lancet. 2020;396(10253):759-69.

131. Klein A, Lin D, Cremer P, et al. Efficacy and safety of rilonacept in recurrent pericarditis: a multicenter phase 2 clinical trial. Circulation. 2019;140(Suppl 1):12851-12851.

132. Klein AL, Imazio M, Cremer P, et al. Phase 3 trial of interleukin-1 trap rilonacept in recurrent pericarditis. N Engl J Med. 2021;384(1):31-41. 